Review Article

\title{
Elephantorrhiza elephantina: Traditional Uses, Phytochemistry, and Pharmacology of an Important Medicinal Plant Species in Southern Africa
}

\author{
Alfred Maroyi \\ Department of Botany, University of Fort Hare, Private Bag X1314, Alice 5700, South Africa \\ Correspondence should be addressed to Alfred Maroyi; amaroyi@ufh.ac.za
}

Received 11 January 2017; Accepted 12 April 2017; Published 14 May 2017

Academic Editor: Sandy van Vuuren

Copyright (C) 2017 Alfred Maroyi. This is an open access article distributed under the Creative Commons Attribution License, which permits unrestricted use, distribution, and reproduction in any medium, provided the original work is properly cited.

\begin{abstract}
Elephantorrhiza elephantina is used in southern Africa as traditional remedy for a wide range of human diseases and ailments including dermatological diseases, gastrointestinal system disorders, sexual dysfunction, sexually transmitted infections, and wounds. The rhizome decoction of E. elephantina is widely used by small-scale farmers in Botswana and South Africa as ethnoveterinary medicine for cattle, goats, horses, pigs, poultry, and sheep. Several classes of phytochemical compounds including anthocyanidins, anthraquinones, esters, fatty acids, phenolic compounds, flavonoids, glycosides, polysterols, saponins, sugars, tannins, and triterpenoids have been isolated from E. elephantina. Scientific studies on E. elephantina indicate that it has a wide range of biological activities including anthelmintic, antibacterial, antifungal, anti-inflammatory and antinociceptive, antiplasmodial, antioxidant, antibabesial, and antirickettsial activities. Elephantorrhiza elephantina is a valuable source of traditional medicine in southern Africa that it is worth additional research attention because of its wide ethnomedicinal applications and promising biological activities. However, the current health-related information on E. elephantina is not sufficiently explored as diverse studies on its chemical and pharmacological activities are required to understand its mechanism of action and to characterize the metabolites responsible for these activities.
\end{abstract}

\section{Introduction}

Elephantorrhiza elephantina (Burch.) Skeels is a member of a small and purely African genus represented by nine species on the continent [1]. Elephantorrhiza elephantina is the type species of the genus, where the generic name "Elephantorrhiza" means "elephant root" and is based, most descriptively, on the large underground stem common to most members of the genus [2]. Four species in this genus, namely, E. burkei Benth., E. elephantina, E. goetzei (Harms) Harms, and E. suffruticosa Schinz, are highly regarded as medicinal plants in southern Africa [3-5]. Elephantorrhiza elephantina is an important plant resource in southern Africa, where it provides food and medicine for the indigenous people and the bark of its tuberous rhizome is a popular source of tanning and dyeing materials [6]. The underground rhizomes, often referred to as roots, are one of the primary herbal medicines in southern Africa. Due to its popularity as herbal medicine, E. elephantina is sold as herbal medicine in the herbal medicine (muthi) markets in Botswana and Zimbabwe [7], the Eastern Cape province of South Africa $[8,9]$, Gauteng province [10], Limpopo province [11], and the Northern Cape province [12]. According to Dold and Cocks [8], the average price of E. elephantina per $\mathrm{kg}$ in the Eastern Cape province is R30.80 (US\$3.60) and $108.80 \mathrm{~kg}$ is the mean quantity sold per trader per year. Due to high demand of the species as herbal medicine and also because harvesters mainly target the rhizomes, E. elephantina is recorded in the Red Data List of Lesotho as data deficient by Talukdar [13] based on the utilization of the species as herbal medicine for stopping bleeding, treating syphilis and intestinal disorders. van Wyk [14] listed E. elephantina as a plant species of high importance with its roots having potential in the formulation of commercial herbal medicines as antioxidant, skin ailments, diarrhoea, perforated ulcers, prostrate hypertrophy, and male pattern baldness in South 
Africa. In South Africa, E. elephantina is used as a traditional remedy for a wide range of ailments, including diarrhoea and dysentery, stomach disorders, skin diseases and acne, haemorrhoids, and perforated peptic ulcers and as emetics [15]. Rhizomes or bark of E. elephantina is crushed with some water added; the resulting paste is applied to hides to tan and dye them a reddish colour [16]. The young shoots of $E$. elephantina are eaten by livestock and its seeds have a sweetish taste followed by a burning sensation and are often roasted in southern Africa as a coffee substitute [16].

With the increasing realization worldwide that traditional medicines based on indigenous medical systems are potential sources of natural products that can be developed into pharmaceutical drugs and health products [14], substantial efforts have been made to investigate ethnomedicinal uses, chemical constituents, and biological activities of E. elephantina during the last three decades. Unfortunately, no comprehensive review on this important plant species in southern Africa has been published, documenting the species' biology, traditional uses, phytochemistry, and pharmacological properties. Therefore, in this study, the advances in traditional utilization, botany, phytochemistry, pharmacology, and safety aspects of E. elephantina are systematically reviewed. In addition to this, the perspectives for the future research on E. elephantina are also discussed in the hope that the article will provide a better understanding of the plant species.

\section{Methodology of the Review}

The literature search was performed from March 2016 to January 2017 using electronic search engines such as Google and Google Scholar and publishing sites such as Elsevier, Science Direct, BioMed Central (BMC), and PubMed. The databases and literature sources were chosen based on the topics covered (i.e., biological activities, ethnobotany, ethnomedicinal uses, ethnopharmacology, pharmacology, phytochemistry, and therapeutic value) and geographical coverage (i.e., southern Africa). The following keywords were used to search literature sources: Acacia elephantina, Elephantorrhiza burchellii and Elephantorrhiza elephantina, dwarf elephant's root, eland's bean, eland's wattle, and elephant's foot. Other literature sources included papers published in international journals, reports from international, regional, and national organizations, conference papers, books, theses, websites, and other grey literature. References were also identified by searching the library collections of the National Herbarium and Botanic Gardens (SRGH), Harare, Zimbabwe, and the University of Fort Hare, South Africa.

\section{Species Description}

Elephantorrhiza elephantina has been recorded in southern Africa, that is, Namibia, Botswana, Zimbabwe, Mozambique, Swaziland, Lesotho, and South Africa. Elephantorrhiza elephantina is usually widespread, often gregarious and forming huge patches in hot and dry areas in grasslands and open scrub [6]. Synonyms of E. elephantina are Acacia elephantina Burch. and Elephantorrhiza burchellii Benth. Elephantorrhiza elephantina is a perennial suffrutex or low shrub, producing annual stems up to $90 \mathrm{~cm}$ tall at ground level, from the woody end of an elongate, with often thickened rhizome up to $8 \mathrm{~m}$ long [16]. Its leaves are alternate, bipinnately compound, almost glabrous with a petiole up to $8 \mathrm{~cm}$ long [17]. The leaves consist of 2-4 pairs of pinnae in lower leaves and 7-17 pairs in upper ones, where the axis is up to $10 \mathrm{~cm}$ long. The leaflets are up to 55 pairs per pinna, linear to oblong in shape, $4-15 \mathrm{~mm}$ long and $0.50-2.50 \mathrm{~mm}$ wide with an asymmetric base, apex acute, and usually mucronate [17]. Elephantorrhiza elephantina inflorescence is an axillary raceme, usually confined to the lower part of the stem usually solitary or clustered. The flowers are bisexual with redbrown glands at the base and free petals which are slightly connate at the base. The petals are linear-oblong, 2-4 mm long and about $1 \mathrm{~mm}$ wide, and yellow-white in colour [16]. The stamens are 10 which are free, with filaments up to $6.50 \mathrm{~mm}$ long [17]. The fruit is a compressed-oblong, straight or slightly curved pod $5-21 \mathrm{~cm}$ long and $3-6 \mathrm{~cm}$ wide, redbrown in colour, prominently transversely veined, and often swollen over the seeds [16].

\section{Vernacular Names of Elephantorrhiza elephantina}

Elephantorrhiza elephantina is known by several vernacular names in its geographical areas of occurrence (Table 1). Literature survey showed no fewer than 41 common or vernacular names for E. elephantina in the seven countries where it is indigenous (Table 1). Local people rarely name plant species that they do not use [18]. This list of common or vernacular names implies that local people in southern Africa have an active interest in E. elephantina. South Africa has the highest number of common or vernacular names (21 in total) followed by Botswana (seven), Namibia (five), and Zimbabwe with four names and the rest of the countries have either one or two names (Table 1). A vernacular name often describes some characteristic feature of the plant species or the plant parts, for example, "eland's bean" (an eland is an indigenous gazelle species); "elandsboontjie"; "eland's wattle"; "elephant's foot"; "elephant-root"; or "dwarf elephant's root" (Table 1). Elephantorrhiza elephantina is commonly referred to as "elandsboontjie" in Afrikaans in South Africa and "eland's bean" and "eland's wattle" in English in Namibia and South Africa because elands feed on the species foliage and pods [19]. The other English common names, "elephant's foot" and "elephant-root," are in reference to large and long rhizomes or roots of the species measuring up to $8 \mathrm{~m}$ long [2]. The common name "dwarf elephant's root" is in reference to the height of E. elephantina, which rarely exceeds one metre in height $[17,20]$ in comparison to a closely related species E. goetzei also known as "elephant's root" but averaging seven metres in height $[5,6]$.

\section{Ethnomedicinal Uses of Elephantorrhiza elephantina}

The rhizome, roots, leaves, and stems of E. elephantina are reported to possess diverse medicinal properties and are used to treat or manage various human and animal ailments 
TABLE 1: Vernacular names of Elephantorrhiza elephantina.

\begin{tabular}{|c|c|c|}
\hline Vernacular name(s), ethnic group or geographical region in brackets & Country & References \\
\hline $\begin{array}{l}\text { Elephant's foot (English), chizezana, mosibe, mosidi, mositsane, } \\
\text { mositsane tjizezana, motshijane (Setswana) }\end{array}$ & Botswana & {$[21-23]$} \\
\hline Mositsane (Sotho) & Lesotho & {$[24]$} \\
\hline Xivurayi (Changana), dwarf elephant's root (English) & Mozambique & {$[25]$} \\
\hline $\begin{array}{l}\text { Elandsboontjie (Afrikaans), eland’s bean (English), gerbwürzel } \\
\text { (German), \|an } \ddagger g \hat{a} b \text { (Khoekhoegowab), omundjoze (Otjiherero) }\end{array}$ & Namibia & {$[26]$} \\
\hline $\begin{array}{l}\text { Baswortel, elandsboontjie, leerbossie, looiersboontjie, olifantswortel } \\
\text { (Afrikaans), dwarf elephant's root, eland's bean, eland's wattle, } \\
\text { elephant's foot, (English), lešhitšana, mosehlana, mošitšana, } \\
\text { motshitshane (Sepedi), gwejobomvu, mositsane (Sotho, Tswana), } \\
\text { ntolwane (Swazi), intolwane, xixuvari (Xhosa), intolwane, intolwanu } \\
\text { (-enkulu), ugweje, umdabu (Zulu) }\end{array}$ & South Africa & $\begin{array}{c}{[4,8,11,17} \\
20,27-33]\end{array}$ \\
\hline Intolwane (Swazi) & Swaziland & {$[34]$} \\
\hline $\begin{array}{l}\text { Elephant-root (English), intolwane encinyane (Ndebele), chizezepasi, } \\
\text { mupangara (Shona) }\end{array}$ & Zimbabwe & {$[20,35]$} \\
\hline
\end{tabular}

and diseases throughout its distributional range in southern Africa (Table 2). A total of 42 and 14 human and animal ailments and diseases, respectively, are treated by herbal medicines prepared from E. elephantina (Table 2). These reports are from all the countries where E. elephantina is indigenous. The country with the highest ethnomedicinal uses is South Africa (45) based on 25 literature records, followed by Lesotho with ten uses and two literature records, Botswana with nine uses and four literature records, Mozambique and Zimbabwe with five uses and two literature records each, and Namibia and Swaziland with a single use and literature record each. Elephantorrhiza elephantina is mainly used to treat disorders of the gastrointestinal tract (21 citations in six countries), followed by veterinary medicine (14 citations in two countries), skin diseases (six citations from South Africa only), pain (five citations in five countries), and infertility and impotence (five citations in four countries). These records show high degree of consensus for the major diseases and ailments (Table 2) and imply high cross-cultural agreement among ethnomedicinal uses of E. elephantina throughout its distributional range.

The rhizome or root decoction of E. elephantina is used to relieve abdominal pains in Lesotho and Zimbabwe $[3,24]$ and chest pains in South Africa [42] and applied to open wounds to stop bleeding [39]. In South Africa, roots and rhizomes of E. elephantina are boiled in water for external use to treat acne and other skin diseases [36-38] while roots and rhizomes of E. elephantina in combination with Pentanisia prunelloides (Klotzsch \& Eckl. \& Zeyh.) Walp. are used to treat eczema [36, 37]. Roots or rhizome decoction of E. elephantina is taken orally as remedy for various ailments and diseases including anemia in Mozambique [25], blood pressure, clearing air canal, erectile dysfunction, haemorrhoids, itching, kidney failure, intestinal disorders, menstrual disorders, peptic ulcers, rheumatic conditions, shingles, sores, syphilis, and tonsillitis in South Africa [4, $12,15,31,38,44,47,48,50]$. In Botswana, rhizome or root powder of E. elephantina is used to wipe the anus of children with bloody diarrhoea, to clean the womb after abortion, as remedy for early menstruating children, earache, erectile dysfunction, and sexually transmitted infections [21, $40,41]$. In Lesotho, rhizome decoction of E. elephantina is used to cleanse blood, as remedy for breast cancer, herpes, infertility, intestinal disorders, stomach problems, syphilis, and tuberculosis [24, 39]. Leaf, rhizome, and root decoction of E. elephantina are used as remedy for diarrhoea and dysentery in Mozambique [45] and South Africa [15, 28, 29, $32,38,44,46]$. In some cases in South Africa, the rhizome of E. elephantina is mixed with Acokanthera oblongifolia Benth. \& Hook.f. ex B.D. Jacks root as remedy for diarrhoea and stomach ailments in South Africa [44]. In Mozambique, root decoction of E. elephantina is taken orally as a pain killer [25] and for sexually transmitted infections [45].

Elephantorrhiza elephantina root decoction is taken orally as emetics for fever in Mozambique [25] and South Africa [42]. The rhizome of E. elephantina is mixed with roots of Pentanisia prunelloides and taken orally as remedy for fever and stomach ailments in Zimbabwe [43]. Elephantorrhiza elephantina is an ingredient of a herbal mixture known as "Sejeso" (Ingwe ${ }^{\circledR}$ brand) made up of Alepidea amatymbica Eckl. \& Zeyh., Hypoxis obtusa Burch. ex Ker Gawl., Pentanisia prunelloides, deionized water, and potassium sorbate as preservative used as remedy for constipation, heartburn, indigestion, loss of appetite, stomach ailments, and vomiting [43]. According to Semenya et al. [49], the rhizome of E. elephantina is mixed with roots of Boscia albitrunca (Burch.) Gilg \& Gilg-Ben., Peltophorum africanum Sond., and Plectranthus ciliatus E. Mey. as remedy for HIV/AIDS opportunistic infections. Research by de Wet et al. [51] revealed that E. elephantina root decoction is taken orally in combination with Cladostemon kirkii (Oliv.) Pax \& Gilg (roots), Drimia delagoensis (Baker) Jessop (bulb), Sarcophyte sanguinea Sparm. ssp. piriei (Hutch.) B. Hansen (bark), and Ranunculus multifidus Forssk. (whole plant) as remedy for shingles. Research by de Wet et al. [51] also revealed that E. elephantina root decoction is taken orally in combination with Cladostemon kirkii (root), Drimia delagoensis (bulb), Ficus sur Forssk. (bark), Ranunculus multifidus (whole plant), 
TABLE 2: Ethnomedicinal uses of Elephantorrhiza elephantina in southern Africa.

\begin{tabular}{|c|c|c|c|}
\hline Use & $\begin{array}{l}\text { Plant part(s) used and } \\
\text { preparation }\end{array}$ & Country of practice & References \\
\hline Abdominal pains & $\begin{array}{l}\text { Rhizome, root decoction } \\
\text { taken orally }\end{array}$ & Lesotho; Zimbabwe & {$[3,24]$} \\
\hline Acne & $\begin{array}{l}\text { Rhizome, root infusion } \\
\text { applied externally }\end{array}$ & South Africa & {$[15,36-38]$} \\
\hline Anemia & Root decoction taken orally & Mozambique & [25] \\
\hline Aphrodisiac & Root decoction taken orally & Zimbabwe & {$[3]$} \\
\hline Bleeding & $\begin{array}{l}\text { Root decoction applied on } \\
\text { affected body part }\end{array}$ & Lesotho & [39] \\
\hline $\begin{array}{l}\text { Bloody diarrhoea in } \\
\text { children }\end{array}$ & $\begin{array}{l}\text { Root powder wiped around } \\
\text { anus }\end{array}$ & Botswana & {$[40,41]$} \\
\hline Blood pressure & $\begin{array}{c}\text { Rhizome decoction taken } \\
\text { orally }\end{array}$ & South Africa & {$[12]$} \\
\hline Breast cancer & $\begin{array}{c}\text { Rhizome decoction taken } \\
\text { orally }\end{array}$ & Lesotho & {$[24]$} \\
\hline Chest pains & Roots taken as emetics & South Africa & {$[42]$} \\
\hline Cleans blood & $\begin{array}{l}\text { Rhizome decoction taken } \\
\text { orally }\end{array}$ & Lesotho & {$[24]$} \\
\hline $\begin{array}{l}\text { Cleaning the womb } \\
\text { after abortion }\end{array}$ & $\begin{array}{c}\text { Rhizome decoction taken } \\
\text { orally }\end{array}$ & $\begin{array}{c}\text { Botswana; South } \\
\text { Africa }\end{array}$ & {$[36,40,41]$} \\
\hline Clearing air canal & $\begin{array}{c}\text { Rhizome decoction taken } \\
\text { orally }\end{array}$ & South Africa & {$[12]$} \\
\hline $\begin{array}{l}\text { Constipation, } \\
\text { heartburn, } \\
\text { indigestion, loss of } \\
\text { appetite, stomach } \\
\text { ailments, vomiting }\end{array}$ & $\begin{array}{c}\text { Ingredient of a herbal } \\
\text { mixture known as "Sejeso" } \\
\text { (Ingwe brand) which also } \\
\text { includes Alepidea } \\
\text { amatymbica Eckl. \& Zeyh., } \\
\text { Hypoxis obtusa Burch. ex } \\
\text { Ker Gawl., Pentanisia } \\
\text { prunelloides (Klotzsch \& } \\
\text { Eckl. \& Zeyh.) Walp., } \\
\text { deionized water and } \\
\text { potassium sorbate as } \\
\text { preservative }\end{array}$ & South Africa & [43] \\
\hline Diarrhoea & $\begin{array}{l}\text { Leaf, rhizome, root, stem } \\
\text { decoction taken orally }\end{array}$ & $\begin{array}{l}\text { Mozambique, South } \\
\text { Africa, Swaziland }\end{array}$ & $\begin{array}{l}{[15,28,29,32} \\
34,38,44-46]\end{array}$ \\
\hline Diarrhoea & $\begin{array}{c}\text { Rhizome mixed with root } \\
\text { of Acokanthera oblongifolia } \\
\text { Benth. \& Hook.f. ex B.D. } \\
\text { Jacks }\end{array}$ & South Africa & {$[44]$} \\
\hline Dysentery & Root decoction taken orally & South Africa & {$[15,38,46]$} \\
\hline Earache & $\begin{array}{l}\text { Rhizome decoction taken } \\
\text { orally }\end{array}$ & Botswana & [41] \\
\hline Eczema & $\begin{array}{l}\text { Roots and rhizome used in } \\
\text { combination with } \\
\text { Pentanisia prunelloides to } \\
\text { treat eczema }\end{array}$ & South Africa & {$[36,37]$} \\
\hline Erectile dysfunction & $\begin{array}{l}\text { Rhizome, root decoction } \\
\text { taken orally }\end{array}$ & $\begin{array}{c}\text { Botswana, South } \\
\text { Africa }\end{array}$ & {$[21,31,47]$} \\
\hline Fever & Roots taken as emetics & $\begin{array}{c}\text { Mozambique, South } \\
\text { Africa }\end{array}$ & {$[25,42]$} \\
\hline
\end{tabular}


TABLE 2: Continued.

\begin{tabular}{|c|c|c|c|}
\hline Use & $\begin{array}{l}\text { Plant part(s) used and } \\
\text { preparation }\end{array}$ & Country of practice & References \\
\hline Fever & $\begin{array}{l}\text { Rhizome decoction taken } \\
\text { orally mixed with } \\
\text { Pentanisia prunelloides }\end{array}$ & Zimbabwe & {$[43]$} \\
\hline Haemorrhoids & $\begin{array}{l}\text { Rhizome, root decoction } \\
\text { taken orally }\end{array}$ & Lesotho, South Africa & {$[15,24,38,48]$} \\
\hline Herpes & $\begin{array}{c}\text { Rhizome decoction taken } \\
\text { orally }\end{array}$ & Lesotho & {$[24]$} \\
\hline $\begin{array}{l}\text { HIV/AIDS } \\
\text { opportunistic diseases }\end{array}$ & $\begin{array}{l}\text { Rhizome decoction taken } \\
\text { orally mixed with roots of } \\
\text { Boscia albitrunca (Burch.) } \\
\text { Gilg \& Gilg-Ben., } \\
\text { Peltophorum africanum } \\
\text { Sond. and Plectranthus } \\
\text { ciliatus E. Mey. }\end{array}$ & South Africa & [49] \\
\hline Itching & $\begin{array}{l}\text { Rhizome decoction taken } \\
\text { orally }\end{array}$ & South Africa & {$[12]$} \\
\hline Infertility in women & $\begin{array}{l}\text { Rhizome, root decoction } \\
\text { taken orally }\end{array}$ & Lesotho, Zimbabwe & {$[3,24]$} \\
\hline Intestinal disorders & $\begin{array}{l}\text { Rhizome, root decoction } \\
\text { taken orally }\end{array}$ & Lesotho, South Africa & {$[15,24,38,39]$} \\
\hline Kidney failure & $\begin{array}{c}\text { Rhizome decoction taken } \\
\text { orally }\end{array}$ & South Africa & {$[12]$} \\
\hline Love charms & Roots taken as emetics & South Africa & {$[42]$} \\
\hline Menstrual problems & $\begin{array}{c}\text { Root, stem decoction taken } \\
\text { orally }\end{array}$ & $\begin{array}{c}\text { Botswana, South } \\
\text { Africa }\end{array}$ & {$[40,44]$} \\
\hline Pain killer & Root decoction taken orally & Mozambique & {$[25]$} \\
\hline Peptic ulcers & Root decoction taken orally & South Africa & {$[4]$} \\
\hline $\begin{array}{l}\text { Rheumatic heart } \\
\text { conditions }\end{array}$ & Root decoction taken orally & South Africa & {$[4]$} \\
\hline $\begin{array}{l}\text { Rheumatic heart } \\
\text { conditions }\end{array}$ & Root decoction taken orally & South Africa & {$[4]$} \\
\hline $\begin{array}{l}\text { Sexually transmitted } \\
\text { infections }\end{array}$ & $\begin{array}{l}\text { Rhizome decoction taken } \\
\text { orally }\end{array}$ & $\begin{array}{l}\text { Botswana, } \\
\text { Mozambique }\end{array}$ & {$[41,45]$} \\
\hline Shingles & $\begin{array}{l}\text { Rhizome decoction taken } \\
\text { orally }\end{array}$ & South Africa & {$[50]$} \\
\hline Shingles & $\begin{array}{c}\text { Root decoction taken orally } \\
\text { in combination with } \\
\text { Cladostemon kirkii (Oliv.) } \\
\text { Pax \& Gilg (roots), Drimia } \\
\text { delagoensis (Baker) Jessop } \\
\text { (bulb), Sarcophyte } \\
\text { sanguinea Sparm. subsp. } \\
\text { piriei (Hutch.) B. Hansen } \\
\text { (bark)and Ranunculus } \\
\text { multifidus Forssk. (whole } \\
\text { plant) }\end{array}$ & South Africa & {$[51]$} \\
\hline Sores & $\begin{array}{l}\text { Rhizome decoction taken } \\
\text { orally }\end{array}$ & South Africa & {$[50]$} \\
\hline Sores & $\begin{array}{c}\text { Root decoction taken orally } \\
\text { in combination with } \\
\text { Cladostemon kirkii (root), } \\
\text { Drimia delagoensis (bulb), } \\
\text { Ficus sur Forssk. (bark), } \\
\text { Ranunculus multifidus } \\
\text { (whole plant), Sarcophyte } \\
\text { sanguinea subsp. piriei and } \\
\text { Senecio serratuloides DC. } \\
\text { (leaves) }\end{array}$ & South Africa & {$[51]$} \\
\hline
\end{tabular}


TABLE 2: Continued.

\begin{tabular}{|c|c|c|c|}
\hline Use & $\begin{array}{l}\text { Plant part(s) used and } \\
\text { preparation }\end{array}$ & Country of practice & References \\
\hline Stomach ailments & Roots taken as emetics & Lesotho, South Africa & {$[24,42]$} \\
\hline Stomach ailments & $\begin{array}{l}\text { Rhizome decoction taken } \\
\text { orally mixed with } \\
\text { Acokanthera oblongifolia } \\
\text { root or Pentanisia } \\
\text { prunelloides }\end{array}$ & $\begin{array}{l}\text { South Africa; } \\
\text { Zimbabwe }\end{array}$ & {$[43,44]$} \\
\hline Sunburn & $\begin{array}{l}\text { Underground parts used to } \\
\text { treat sunburn }\end{array}$ & South Africa & {$[15,38]$} \\
\hline Syphilis & Root decoction taken orally & Lesotho, South Africa & {$[15,24,38,39]$} \\
\hline Tonsillitis & $\begin{array}{l}\text { Rhizome boiled and extract } \\
\text { taken orally }\end{array}$ & South Africa & {$[12]$} \\
\hline Tuberculosis & $\begin{array}{c}\text { Rhizome decoction taken } \\
\text { orally }\end{array}$ & Lesotho & {$[24]$} \\
\hline \multicolumn{4}{|l|}{$\begin{array}{l}\text { Ethnoveterinary } \\
\text { medicine }\end{array}$} \\
\hline Appetite stimulant & Rhizome decoction & South Africa & [27] \\
\hline Black quarter & Rhizome decoction & South Africa & {$[27,30]$} \\
\hline Cough & Rhizome decoction & South Africa & {$[52]$} \\
\hline Diarrhoea & Rhizome decoction & South Africa & {$[30,48,52]$} \\
\hline $\begin{array}{l}\text { Dysentery in cattle } \\
\text { and horses }\end{array}$ & Root decoction & South Africa & {$[48]$} \\
\hline $\begin{array}{l}\text { Ectoparasites in goats } \\
\text { (mites, ticks) }\end{array}$ & Root decoction & South Africa & {$[33]$} \\
\hline $\begin{array}{l}\text { Gastrointestinal } \\
\text { parasites }\end{array}$ & Rhizome decoction & South Africa & {$[30]$} \\
\hline Gall sickness & Rhizome decoction & South Africa & {$[30]$} \\
\hline Heartwater & Rhizome decoction & South Africa & {$[27,52]$} \\
\hline Mange & $\begin{array}{l}\text { Root decoction given to } \\
\text { cows }\end{array}$ & South Africa & {$[53]$} \\
\hline Pneumonia & Rhizome decoction & South Africa & {$[52]$} \\
\hline $\begin{array}{l}\text { Retained placenta in } \\
\text { cattle }\end{array}$ & Rhizome decoction & $\begin{array}{c}\text { Botswana, South } \\
\text { Africa }\end{array}$ & {$[22,30]$} \\
\hline Tonic & Rhizome decoction & South Africa & [27] \\
\hline
\end{tabular}

Sarcophyte sanguinea ssp. piriei, and Senecio serratuloides DC. (leaves) as remedy for sores.

Rhizome decoction of E. elephantina is widely used by small-scale farmers in Botswana and South Africa as ethnoveterinary medicine for poultry and retained placenta in cattle and as ethnoveterinary medicine for other animals such as goats, horses, pigs, and sheep and for diseases such as black quarter, appetite stimulant, coughing, diarrhoea, gastrointestinal parasites, gall sickness, heartwater, mange, pneumonia, and ectoparasites [22, 23, 27, 30, 33, 48, 52, 53]. The young shoots of E. elephantina are eaten by livestock and wild animals in southern Africa [6]. In Namibia, the pods of E. elephantina are eaten by both people and animals [26].

\section{Phytochemistry}

Multiple classes of phytochemicals including anthocyanidins, anthraquinones, esters, fatty acids, phenolic compounds, flavonoids, glycosides, polysterols, saponins, sugars, tannins, and triterpenoids have been isolated from rhizome extracts of E. elephantina [41,54-57]. Considerable pharmacological potential of E. elephantina has been documented through detection, isolation and purification of its natural products via advances in spectrometric techniques such as attenuated total reflection (ATR), Fourier transform infrared (FTIR) spectroscopy, liquid chromatography electron spray ionization mass spectroscopy (LC-ESI-MS), gas chromatographymass spectrometry (GC-MS), and nuclear magnetic resonance (NMR) for structural elucidation of new and complex compounds (Table 3). Advanced research through ATR, LCESI-MS, FTIR, GC-MS, and NMR spectroscopy enabled researchers to have a better understanding of the correlations between molecular conformation and biological activities of the natural compounds of E. elephantina and its importance as herbal medicine. The compounds isolated from E. elephantina are documented and listed in Table 3 and their structures are displayed in Figure 1. Aaku et al. [41] isolated the following compounds from n-butanol 
TABLE 3: Phytochemical compounds isolated from rhizomes or roots of Elephantorrhiza elephantina.

\begin{tabular}{|c|c|c|c|}
\hline $\begin{array}{l}\text { Phytochemical } \\
\text { compounds }\end{array}$ & Extract & $\begin{array}{l}\text { Method of compound } \\
\text { characterization }\end{array}$ & References \\
\hline \multicolumn{4}{|l|}{ Anthraquinone } \\
\hline Anthraquinone 38 & $\begin{array}{l}\text { Chloroform, } \\
\text { methanol }\end{array}$ & LC-ESI-MS & {$[54]$} \\
\hline \multicolumn{4}{|l|}{ Ester } \\
\hline Ethyl gallate 4 & n-butanol & GC-MS & {$[41]$} \\
\hline Butanedioic acid 19 & Hexane & GC-MS & {$[55]$} \\
\hline Benzoic acid 20 & Hexane & GC-MS & {$[55]$} \\
\hline $\begin{array}{l}\text { 3-phenyl-2-propenoic } \\
\text { acid } 21\end{array}$ & Hexane & GC-MS & {$[55]$} \\
\hline Nonanedioic acid 22 & Hexane & GC-MS & {$[55]$} \\
\hline $\begin{array}{l}\text { Methyl 3-(3,5-di-tert- } \\
\text { butyl-4-hydroxy- } \\
\text { phenyl)propionate } \\
27\end{array}$ & Hexane & GC-MS & {$[55]$} \\
\hline \multicolumn{4}{|l|}{ Fatty acid } \\
\hline Hexadecanoic acid 15 & Hexane & GC-MS & {$[55]$} \\
\hline 9,12-Octadecadienoic $\mathbf{1 6}$ & Hexane & GC-MS & {$[55]$} \\
\hline 9-Octadecenoic $\mathbf{1 7}$ & Hexane & GC-MS & {$[55]$} \\
\hline Octadecanoic acid $\mathbf{1 8}$ & Hexane & GC-MS & {$[55]$} \\
\hline Tridecanoic acid 23 & Hexane & GC-MS & {$[55]$} \\
\hline $\begin{array}{l}\text { Methyl pentadecanoate } \\
\mathbf{2 4}\end{array}$ & Hexane & GC-MS & {$[55]$} \\
\hline $\begin{array}{l}\text { Methyl } \\
\text { hexadec-9-enoate } 25\end{array}$ & Hexane & GC-MS & {$[55]$} \\
\hline $\begin{array}{l}\text { Methyl hexadecanoate } \\
26\end{array}$ & Hexane & GC-MS & {$[55]$} \\
\hline $\begin{array}{l}\text { Cis-10-Heptadecenoic } \\
\text { acid } \mathbf{2 8}\end{array}$ & Hexane & GC-MS & {$[55]$} \\
\hline $\begin{array}{l}\text { Methyl heptadecanoate } \\
29\end{array}$ & Hexane & GC-MS & {$[55]$} \\
\hline Methyl octadecanoate 30 & Hexane & GC-MS & {$[55]$} \\
\hline $\begin{array}{l}\text { Cis-5,8,11,14,17- } \\
\text { eicosapenta-enoic acid } \\
31\end{array}$ & Hexane & GC-MS & {$[55]$} \\
\hline Eicosanoic acid 32 & Hexane & GC-MS & {$[55]$} \\
\hline Methyl tetracosanoate 33 & Hexane & GC-MS & {$[55]$} \\
\hline Pentacosanoic acid $\mathbf{3 4}$ & Hexane & GC-MS & {$[55]$} \\
\hline Hexacosanoic acid 35 & Hexane & GC-MS & {$[55]$} \\
\hline Methyl octacosanoate 36 & Hexane & GC-MS & {$[55]$} \\
\hline Tetradecanedioic acid 37 & Hexane & GC-MS & {$[55]$} \\
\hline \multicolumn{4}{|l|}{ Flavonoids } \\
\hline Dihydrokaempferol 1 & n-butanol & GC-MS & {$[41]$} \\
\hline Kaempferol 2 & $\begin{array}{l}\text { Ethanol, } \\
\text { n-butanol }\end{array}$ & GC-MS, LC-ESI-MS & {$[41,56]$} \\
\hline \multicolumn{4}{|l|}{ Glycoside } \\
\hline $\begin{array}{l}\text { Ethyl-1-O- } \beta \text {-D- } \\
\text { galactopyranoside } \\
8\end{array}$ & n-butanol & GC-MS & {$[41]$} \\
\hline
\end{tabular}


TABle 3: Continued.

\begin{tabular}{|c|c|c|c|}
\hline $\begin{array}{l}\text { Phytochemical } \\
\text { compounds }\end{array}$ & Extract & $\begin{array}{c}\text { Method of compound } \\
\text { characterization }\end{array}$ & References \\
\hline \multicolumn{4}{|l|}{ Phenolic compounds } \\
\hline $\begin{array}{l}\text { 2-(3,4- } \\
\text { Dihydroxyphenyl) } \\
\text { ethanol } 6\end{array}$ & n-butanol & GC-MS & {$[41]$} \\
\hline Catechin 3 & $\begin{array}{l}\text { Chloroform, } \\
\text { methanol, } \\
\text { n-butanol }\end{array}$ & GC-MS, NMR & {$[41,57]$} \\
\hline Gallic acid 5 & $\begin{array}{l}\text { Chloroform, } \\
\text { methanol, } \\
\text { n-butanol }\end{array}$ & GC-MS, NMR & {$[41,57]$} \\
\hline $\begin{array}{l}\text { 4-Hydroxybenzoic acid } \\
7\end{array}$ & n-butanol & GC-MS & {$[41]$} \\
\hline $\begin{array}{l}\text { Quercetin 3-O- } \beta \text {-D- } \\
\text { glucopyranoside } \\
\mathbf{9}\end{array}$ & $\begin{array}{l}\text { Chloroform, } \\
\text { methanol, } \\
\text { n-butanol }\end{array}$ & GC-MS, NMR & {$[41,57]$} \\
\hline $\begin{array}{l}\text { Epigallocatechin gallate } \\
\mathbf{4 4}\end{array}$ & Ethanol & LC-ESI-MS & {$[56]$} \\
\hline Quercetin 45 & Ethanol & LC-ESI-MS & {$[56]$} \\
\hline Epicatechin gallate 46 & Ethanol & LC-ESI-MS & {$[56]$} \\
\hline Methyl gallate $\mathbf{1 0}$ & $\begin{array}{l}\text { Chloroform, } \\
\text { methanol }\end{array}$ & NMR & {$[57]$} \\
\hline $\begin{array}{l}\text { 3-O-Galloyl-3,3' }, 5,5^{\prime}, 7- \\
\text { pentahydroxyflavone } \\
\mathbf{1 2}\end{array}$ & $\begin{array}{l}\text { Chloroform, } \\
\text { methanol }\end{array}$ & NMR & {$[57]$} \\
\hline $\begin{array}{l}\text { Taxifolin- } 3^{\prime}-\mathrm{O}-\beta-\mathrm{D}- \\
\text { glucoside } \\
\mathbf{1 3}\end{array}$ & $\begin{array}{l}\text { Chloroform, } \\
\text { methanol }\end{array}$ & NMR & {$[57]$} \\
\hline Epicatechin 14 & $\begin{array}{l}\text { Chloroform, } \\
\text { ethanol, } \\
\text { methanol }\end{array}$ & $\begin{array}{l}\text { FTIR, LC-ESI-MS, } \\
\text { NMR }\end{array}$ & {$[56,57]$} \\
\hline \multicolumn{4}{|l|}{ Phytosterols } \\
\hline$\beta$-Sitosterol 11 & $\begin{array}{l}\text { Chloroform, } \\
\text { methanol }\end{array}$ & NMR & {$[57]$} \\
\hline \multicolumn{4}{|l|}{ Saponin } \\
\hline Diosgenin 40 & $\begin{array}{l}\text { Chloroform, } \\
\text { methanol }\end{array}$ & LC-ESI-MS & {$[54]$} \\
\hline \multicolumn{4}{|l|}{ Sugar } \\
\hline Rhamnose 41 & $\begin{array}{l}\text { Chloroform, } \\
\text { methanol }\end{array}$ & LC-ESI-MS & {$[54]$} \\
\hline Glucuronic acid 42 & $\begin{array}{l}\text { Chloroform, } \\
\text { ethanol, } \\
\text { methanol }\end{array}$ & LC-ESI-MS & {$[54,56]$} \\
\hline Arabinose 43 & $\begin{array}{l}\text { Chloroform, } \\
\text { ethanol, } \\
\text { methanol }\end{array}$ & LC-ESI-MS & {$[54,56]$} \\
\hline \multicolumn{4}{|l|}{ Triterpenoid } \\
\hline Oleanolic acid 39 & $\begin{array}{l}\text { Chloroform, } \\
\text { methanol }\end{array}$ & LC-ESI-MS & {$[54]$} \\
\hline
\end{tabular}


<smiles>O=C1c2c(O)cc(O)cc2O[C@H](c2ccc(O)cc2)[C@@H]1O</smiles><smiles>CCOC(=O)c1cc(O)c(O)c(O)c1</smiles>

Ethyl gallate 4<smiles>O=c1c(O)c(-c2ccc(O)cc2)oc2cc(O)cc(O)c12</smiles>

Kaempferol 2<smiles>O=C(O)c1cc(O)c(O)c(O)c1</smiles>

Gallic acid 5<smiles>Oc1cc(O)c2c(c1)O[C@H](c1ccc(O)c(O)c1)[C@H](O)C2</smiles>

Catechin 3<smiles>OCCc1ccc(O)c(O)c1</smiles>

2-(3,4-Dihydroxyphenyl) ethanol 6<smiles>O=C(O)c1ccc(O)cc1</smiles>

4-Hydroxybenzoic acid 7
Ethyl-1-O- $\beta$-D-galactopyranoside 8<smiles>O=c1c(O[C@@H]2O[C@H](CO)[C@@H](O)[C@H](O)[C@H]2O)c(-c2ccc(O)c(O)c2)oc2cc(O)cc(O)c12</smiles>

Quercetin 3-O- $\beta$-D-glucopyranoside 9<smiles>CC[C@H](CC[C@H](C)[C@H]1CC[C@H]2[C@H]3CC=C4C[C@@H](O)CC[C@]4(C)[C@H]3CC[C@]21C)C(C)C</smiles>

3-O-Galloyl-3,3' ${ }^{\prime}, 5,5^{\prime}, 7$-pentahydroxyflavone 12<smiles>O=C1c2c(O)cc(O)cc2O[C@H](c2ccc(O)c(O[C@@H]3O[C@H](CO)[C@@H](O)[C@H](O)[C@H]3O)c2)[C@@H]1O</smiles>

Taxifolin- $3^{\prime}$-O- $\beta$-D-glucoside 13<smiles>Oc1cc(O)c2c(c1)O[C@H](c1ccc(O)c(O)c1)[C@H](O)C2</smiles>

Epicatechin 14

FIgure 1: Continued. 
<smiles>CCCCCCCCCCCCCCCC(=O)O</smiles>

Hexadecanoic acid 15<smiles>CCCCCC=CCC=CCCCCCCCC(=O)O</smiles>

9,12-Octadecadienoic 16

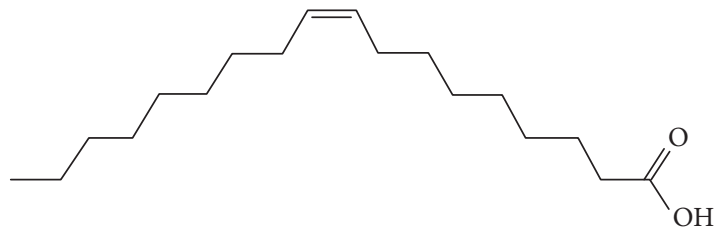

9-Octadecenoic $\mathbf{1 7}$<smiles>CCCCCCCCCCCCCCCCCC(=O)O</smiles>

Octadecanoic acid 18<smiles>O=C(O)CCC(=O)O</smiles>

Butanedioic acid 19<smiles>O=C(O)c1ccccc1</smiles>

Benzoic acid $\mathbf{2 0}$<smiles>O=C(O)/C=C/c1ccccc1</smiles>

3-Phenyl-2-propenoic acid 21<smiles>O=C(O)CCCCCCCC(=O)O</smiles>

Nonanedioic acid 22<smiles>CCCCCCCCCCCCC(=O)O</smiles>

Tridecanoic acid $\mathbf{2 3}$<smiles>CCCCCCCCCCCCCCCC(=O)OC</smiles>

Methyl hexadecanoate $\mathbf{2 6}$

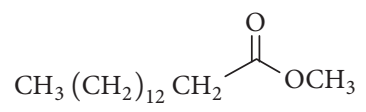

Methyl pentadecanoate $\mathbf{2 4}$<smiles>COC(=O)CCc1cc(C(C)(C)C)c(O)c(C(C)(C)C)c1</smiles>
Methyl 3-(3,5-di-tert-butyl-4-hydroxy-phenyl)

$$
\begin{gathered}
\text { Methyl 3-(3,5-di-tert-butyl-4-hy } \\
\text { propionate } \mathbf{2 7}
\end{gathered}
$$<smiles>CCCCCC/C=C\CCCCCCCC(=O)O</smiles>

Methyl hexadec-9-enoate $\mathbf{2 5}$<smiles>CCCCCCCCCCCCCCC(=O)O</smiles>

Cis-10-heptadecenoic acid $\mathbf{2 8}$<smiles>CCCCCCCCCCCCCCCCC(=O)OC</smiles>

Methyl heptadecanoate 29<smiles>CCCCCCCCCCCCCCCCCC(=O)OC</smiles>

Methyl octadecanoate $\mathbf{3 0}$<smiles>CC/C=C\C/C=C\C/C=C\C/C=C\C/C=C\CCCC(=O)O</smiles>

Cis-5,8,11,14,17-eicosapenta-enoic acid 31<smiles>CCCCCCCCCCCCCCCCCCCCCCCCC(=O)OC</smiles>

Eicosanoic acid 32

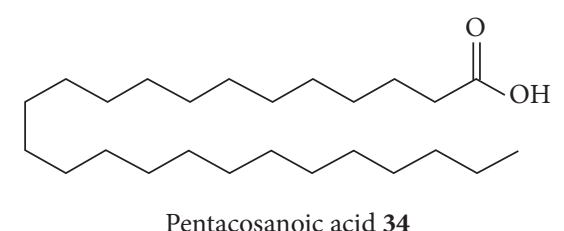

Figure 1: Continued. 
<smiles>CCCCCCCCCCCCCCCCCCCCCCCC(=O)O</smiles>

Hexacosanoic acid 35<smiles>O=C(O)CCCCCCCCCCCCC(=O)O</smiles>

Tetradecanedioic acid 37

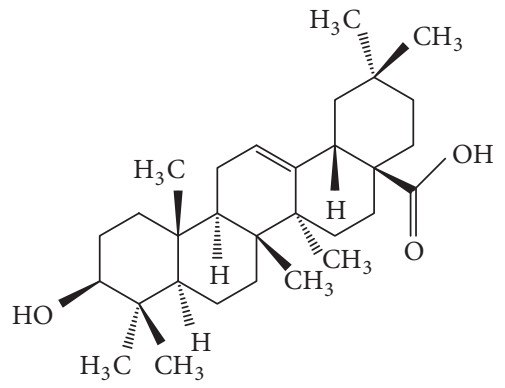

Oleanolic acid 39

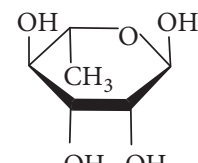

$\mathrm{OH} \mathrm{OH}$

Rhamnose $\mathbf{4 1}$

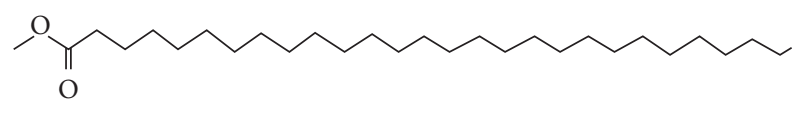

Methyl octacosanoate 36<smiles>O=C1c2ccccc2C(=O)c2ccccc21</smiles>

Anthraquinone 38

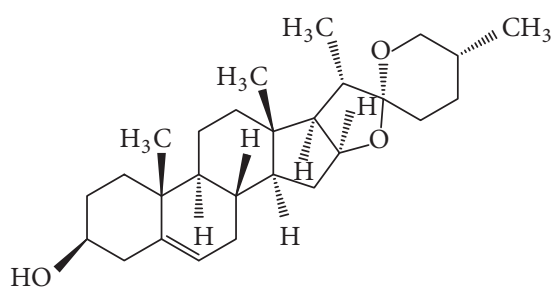

Diosgenin 40

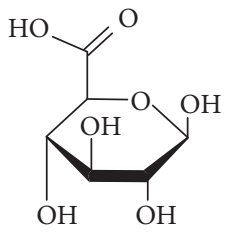

Glucuronic acid $\mathbf{4 2}$

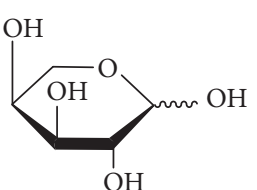

Arabinose $\mathbf{4 3}$<smiles>O=C(O[C@H]1Cc2c(O)cc(O)cc2O[C@@H]1c1cc(O)c(O)c(O)c1)c1cc(O)c(O)c(O)c1</smiles>

Epigallocatechin gallate $\mathbf{4 4}$<smiles>O=c1c(O)c(-c2ccc(O)c(O)c2)oc2cc(O)cc(O)c12</smiles>

Quercetin 45<smiles>O=C(O[C@@H]1Cc2c(O)cc(O)cc2O[C@H]1c1ccc(O)c(O)c1)c1cc(O)c(O)c(O)c1</smiles>

Epicatechin gallate 46

FIGURE 1: Chemical structures of major compounds isolated from rhizomes or roots of Elephantorrhiza elephantina. 
rhizome extracts of E. elephantina: dihydrokaempferol 1, kaempferol 2, (-)-catechin 3, ethyl gallate 4, gallic acid 5, 2-(3,4-dihydroxyphenyl) ethanol 6, 4-hydroxybenzoic acid 7, ethyl-1-O- $\beta$-D-galactopyranoside 8 , and quercetin 3-O- $\beta$ D-glucopyranoside 9. Phytochemical study of E. elephantina rhizomes by Mthembu [57] showed the presence of several phenolic compounds including catechin $\mathbf{3}$, gallic acid $\mathbf{5}$, quercetin 3-O- $\beta$-D-glucopyranoside 9, methyl gallate 10, $\beta$-sitosterol 11, 3-O-galloyl-3,3',5,5',7-pentahydroxyflavone 12, taxifolin- $3^{\prime}-\mathrm{O}-\beta$-D-glucoside 13 , and epicatechin 14. Recently, Msimanga et al. [55] isolated the following compounds from hexane root extracts of E. elephantina: hexadecanoic 15, 9,12-octadecadienoic 16, 9-octadecenoic 17, octadecanoic acid 18, butanedioic acid 19, benzoic acid 20,3phenyl-2-propenoic acid 21, nonanedioic acid 22, tridecanoic acid 23, methyl pentadecanoate $\mathbf{2 4}$, methyl hexadec-9-enoate 25, methyl hexadecanoate 26, methyl 3-(3,5-di-tert-butyl4-hydroxy-phenyl)propionate 27 , cis-10-Heptadecenoic acid 28, methyl heptadecanoate 29, methyl octadecanoate 30, cis-5,8,11,14,17-eicosapenta-enoic acid 31, eicosanoic acid 32, methyl tetracosanoate 33, pentacosanoic acid 34, hexacosanoic acid 35, methyl octacosanoate 36 , and tetradecanedioic acid 37. The phytochemical studies of the rhizome extracts of E. elephantina carried out by Mpofu et al. [54] showed the presence of anthraquinone 38, triterpenoids oleanolic acid 39, diosgenin 40, rhamnose 41, glucuronic acid 42, and arabinose 43. In another phytochemical evaluation of E. elephantina rhizome extracts, Mpofu et al. [56] isolated kaempferol 2, epicatechin 14, glucuronic acid 42, arabinose 43, epigallocatechin gallate 44 , quercetin 45 , and epicatechin gallate 46. The major phytochemical compounds isolated from E. elephantina are mainly fatty acids $(39.13 \%$ of all known compounds isolated from the species), followed by phenolic compounds (26.09\%) and esters (13.04\%) and the contribution of the rest of the compounds is less than $10 \%$ each; see Table 3.

\section{Pharmacological Activities}

A number of pharmacological activities of E. elephantina have been reported in literature corroborating some of the ethnomedicinal uses listed in Table 2. Some of the pharmacological activities of E. elephantina listed in literature include anthelmintic [58-60], antibacterial [21, 28, 41, 43, $50,61]$, antifungal [21, 41, 50, 61], anti-inflammatory and antinociceptive [62], antiplasmodial [63], antioxidant [54], and antibabesial and antirickettsial $[64,65]$ activities.

7.1. Anthelmintic Activity. Maphosa et al. [58] evaluated in vitro anthelmintic activities of crude aqueous extracts of E. elephantina roots on the eggs and larvae of the nematode parasite Haemonchus contortus using Valbazen ${ }^{\circledR}(11.36 \%$ albendazole) at $10 \mathrm{mg} / \mathrm{kg}$ and $0.5 \mathrm{~mL} / \mathrm{kg}$ distilled water as positive and negative controls, respectively. Elephantorrhiza elephantina had $100 \%$ egg hatch inhibition at a concentration as low as $2.5 \mathrm{mg} / \mathrm{mL}$. At the lowest concentration of $0.63 \mathrm{mg} / \mathrm{mL}$ tested, E. elephantina inhibited egg hatching by $>96 \%$ and this was comparable to albendazole at the same concentration [58]. Elephantorrhiza elephantina had complete inhibition of larval development at a concentration of $1.25 \mathrm{mg} / \mathrm{mL}$ [58]. This study by Maphosa et al. [58] demonstrated that inhibition of egg hatching and larval development increased significantly with increasing concentration of E. elephantina root extract. In another study, Maphosa and Masika [59] evaluated efficacy of E. elephantina aqueous root extracts in naturally mixed infections of gastrointestinal worms and Coccidia species in goats that had not been dosed for a period of two months, using Valbazen ( $11.36 \%$ albendazole) at $10 \mathrm{mg} / \mathrm{kg}$ and $0.5 \mathrm{~mL} / \mathrm{kg}$ distilled water as positive and negative controls, respectively. In this study, E. elephantina caused reduction of Trichuris eggs on days 3 and 6 at $250 \mathrm{mg} / \mathrm{kg}$ dose. This study also revealed efficacy of E. elephantina against strongyle and Eimeria spp. at $500 \mathrm{mg} / \mathrm{kg}$. The reduction in faecal egg counts in dosed extracts with E. elephantina against mixed gastrointestinal parasite infections shows that this species possess anthelmintic properties and there is credence in its ethnoveterinary use against gastrointestinal parasites in goats. In another study, Maphosa and Masika [60] evaluated anthelmintic activity of aqueous, hexane, and ethyl root extract of E. elephantina against adult Haemonchus contortus using a bioactivity-guided assay with albendazole and distilled water as positive and negative controls, respectively. The aqueous and ethyl acetate fractions showed high motility inhibition at concentrations of $2.50 \mathrm{mg} / \mathrm{mL}$ and above after 6-hour exposure, while the hexane fraction showed motility inhibition at concentrations of $5 \mathrm{mg} / \mathrm{mL}$ and above. After 30-hour exposure, all the fractions, that is, aqueous, hexane, and ethyl acetate fractions, and albendazole (commercial drug) showed inhibition of motility and the mortality indexes were not significantly different from each other [60]. All the anthelmintic evaluations carried out so far [58-60] confirmed the anthelmintic activities of the root of E. elephantina, a plant species widely used as anthelmintic remedy by small-scale farmers in South Africa.

7.2. Antibacterial Activity. Aaku et al. [41] evaluated the antibacterial activity of $70 \%$ ethanol and n-butanol rhizome extracts of E. elephantina using the thin-layer chromatography (TLC) bioautography technique with chloramphenicol and miconazole as positive and negative controls, respectively. Both extracts showed activity against Bacillus subtilis, Escherichia coli, Pseudomonas aeruginosa, and Staphylococcus aureus at loadings lower than $15 \mu \mathrm{g}$. Among the purified compounds, only ethyl gallate 4 and gallic acid 5 showed activity against Bacillus subtilis and Staphylococcus aureus at loadings lower than $50 \mu \mathrm{g}$. Similar results were obtained by Cueva et al. [66] who assessed the influence of pure phenolic compounds such as catechin 3 , ethyl gallate 4 , gallic acid 5, and epicatechin 14 on the inhibition of the growth of potential respiratory pathogens. These authors found that nonflavonoid compounds such as ethyl gallate $\mathbf{4}$ and gallic acid 5 were more active than flavonoids such as catechin $\mathbf{3}$ and epicatechin 14.

Mathabe et al. [28] evaluated the antibacterial activities of aqueous, acetone, ethanol, and methanol root extracts of $E$. elephantina against bacteria that cause gastrointestinal infections, namely, Staphylococcus aureus, Vibrio cholerae, Shigella dysentery, Shigella sonnei, Shigella flexneri, and Shigella boydii, 
and the minimum inhibitory concentration (MIC) of active extracts was determined by the microplate dilution assay. Mathabe et al. [28] used ten microliters of dimethyl sulfoxide (DMSO) per well as negative control while discs $(5 \mathrm{~mm}$ in diameter) of nalidixic acid (30 mg), erythromycin (15 mg), and cotrimoxazole $(25 \mathrm{mg}$ ) were used as positive controls. MIC activities against the pathogens ranged between 0.08 and $0.63 \mathrm{mg} / \mathrm{mL}$, and the highest inhibition was exhibited against Shigella flexneri with MIC values ranging from 0.08 to $0.16 \mathrm{mg} / \mathrm{mL}$ [28], and these findings somehow confirm the species' antibacterial potential and its usefulness in the treatment and management of gastrointestinal infections. Mukanganyama et al. [21] evaluated antibacterial activities of ethanol root extracts of E. elephantina against Bacillus cereus, Bacillus subtilis, Escherichia coli, Pseudomonas aeruginosa, and Staphylococcus aureus using the agar diffusion assay. The species exhibited antibacterial properties against all microorganisms tested and the authors assessed the minimal inhibitory concentrations (MICs) against Mycobacterium aurum, where E. elephantina showed some activity with MIC value of $1.25 \mathrm{mg} / \mathrm{mL}$ [21].

Mabona et al. [61] evaluated antibacterial activities of aqueous and dichlomethane/methanol (1:1) leaf, root, and rhizome extracts of E. elephantina using the micro-titre plate dilution technique against dermatologically relevant pathogens such as Brevibacillus agri, Propionibacterium acnes, Pseudomonas aeruginosa, Staphylococcus aureus and Staphylococcus epidermidis with ciprofloxacin as positive control and acetone and dimethyl sulfoxide (DMSO) as negative controls. Mabona et al. [61] found varied antibacterial activities of the aqueous and dichlomethane/methanol (1:1) leaf, root and rhizome extracts with minimum inhibition concentration (MIC) ranging from 0.05 to $>16.00 \mathrm{mg} / \mathrm{mL}$. Antibacterial activities were displayed by dichlomethane/methanol leaf, root and rhizome extracts against Propionibacterium acnes (MIC values ranging from 0.05 to $1.00 \mathrm{mg} / \mathrm{mL}$ ), Staphylococcus aureus $(0.50 \mathrm{mg} / \mathrm{mL})$ and Staphylococcus epidermis $(0.38$ to $1.00 \mathrm{mg} / \mathrm{mL})$ as well as aqueous and dichlomethane/methanol root and rhizome extracts against Brevibacillus agri with MIC value of $0.50 \mathrm{mg} / \mathrm{mL}$. The leaf, root and rhizome extracts of E. elephantina are reported to be traditionally used to treat acne vulgaris and pimples and such usage was corroborated by noteworthy activity against Propionibacterium acnes with MIC values between 0.05 and $2.0 \mathrm{mg} / \mathrm{mL}$ [61]. Propionibacterium acnes is an important skin pathogen responsible for the chronic inflammatory disease of the sebaceous glands and hair follicles of the skin [61]. The aqueous root extracts of Pentanisia prunelloides combined $(1: 1)$ with $E$. elephantina displayed synergistic interactions with sum of the fractional inhibitory concentration ( $\Sigma$ FIC) values ranging from 0.31 to $0.38 \mathrm{mg} / \mathrm{mL}$ against Staphylococcus aureus and Staphylococcus epidermidis. The synergistic interactions noted for Pentanisia prunelloides and E. elephantina by Mabona et al. [61] validate their antibacterial effects as these two species are often used in combination as herbal medicines for treating microbial infections in southern Africa. Similarly, Nciki et al. [50] evaluated antibacterial activities of aqueous and dichlomethane/methanol (1:1) root extract of E. elephantina using the micro-titre plate dilution technique against dermatologically relevant pathogens such as Brevibacillus agri, Escherichia coli, Propionibacterium acnes, Pseudomonas aeruginosa, Staphylococcus aureus and Staphylococcus epidermidis with ciprofloxacin as positive control. Best antimicrobial results were demonstrated by dichlomethane/methanol extracts against Escherichia coli with MIC value of $130 \mu \mathrm{g} / \mathrm{mL}$, Brevibacillus agri (MIC value of $250 \mu \mathrm{g} / \mathrm{mL}$ ), Propionibacterium acnes (MIC value of $250 \mu \mathrm{g} / \mathrm{mL}$ ) and Pseudomonas aeruginosa with MIC value of $250 \mu \mathrm{g} / \mathrm{mL}$ [50]. Nciki et al. [50] and Mabona et al. [61] obtained similar results in terms of overall antibacterial activities displayed against Brevibacillus agri, Propionibacterium acnes and Pseudomonas aeruginosa although Nciki et al. [50] also assessed the antibacterial activities of E. elephantina against Escherichia coli. Nciki et al. [50] assessed antibacterial activities of aqueous and dichlomethane/methanol (1:1) root extracts of E. elephantina while Mabona et al. [61] assessed antibacterial activities of other plant parts which included leaves and rhizomes. Therefore, the results obtained by both Nciki et al. [50] and Mabona et al. [61] provide a scientific rational for the traditional use of E. elephantina as herbal medicine against several skin infections in South Africa such as acne $[15,36-38]$, eczema $[36,37]$, itching $[12]$, sores $[50,51]$ and sunburn $[15,38]$.

Mpofu et al. [43] evaluated antibacterial activity of the methanol and aqueous rhizome extracts of E. elephantina using the micro-titre plate dilution technique against Bacillus cereus, Enterococcus faecalis and Escherichia coli with ciprofloxacin as positive control and distilled water and dimethyl sulfoxide (DMSO) as negative controls. The minimum inhibitory concentration (MIC) values for the aqueous ( 0.50 $2.00 \mathrm{mg} / \mathrm{mL})$ and methanol $(0.20-4.00 \mathrm{mg} / \mathrm{mL})$ extracts independently demonstrated varied efficacies depending on the pathogen of study. Mpofu et al. [43] also evaluated the antibacterial activity of E. elephantina with Pentanisia prunelloides combined in 1:1 ratios, displaying synergistic interactions with sum of the fractional inhibitory concentration ( $\Sigma$ FIC) values ranging from 0.19 to $1.00 \mathrm{mg} / \mathrm{mL}$ for aqueous extracts and 0.60 to $0.80 \mathrm{mg} / \mathrm{mL}$ for methanol extracts against Bacillus cereus, Enterococcus faecalis and Escherichia coli. The antibacterial activity of E. elephantina in combination with Pentanisia prunelloides were determined as a validation of their combined use in southern African traditional medicine. Mpofu et al. [43] also evaluated the antibacterial activity of epicatechin $\mathbf{1 4}$ and hexadecanoic acid $\mathbf{1 5}$ isolated from E. elephantina rhizomes using the microtitre plate dilution technique against Bacillus cereus, Enterococcus faecalis and Escherichia coli with ciprofloxacin as positive control and distilled water and dimethyl sulfoxide (DMSO) as negative controls. The efficacy for the two compounds measured via MIC values ranged between 0.13 and $0.63 \mathrm{mg} / \mathrm{mL}$, while synergistic interactions were noted against Escherichia coli and Enterococcus faecalis with ( $\mathrm{FIC}$ ) values of $0.09 \mathrm{mg} / \mathrm{mL}$ and $0.50 \mathrm{mg} / \mathrm{mL}$, respectively [43]. Therefore, the two compounds epicatechin 14 and hexadecanoic acid 15 showed synergistically enhanced activity especially against Escherichia coli and Enterococcus faecalis. Furthermore, previous studies have shown that hexadecanoic acid $\mathbf{1 5}$ is active against various 
bacterial strains [67] including Escherichia coli [68] and epicatechin 14 is also active against Escherichia coli and can play an important role in developing pharmaceutical drugs against urinary tract infections [69]. Epicatechin 14 has also been implicated for antibacterial activity against Escherichia coli, Bacillus cereus, Staphylococcus aureus, and Shigella flexneri at minimum inhibition concentration (MIC) values ranging from 12.50 to $100 \mathrm{mg} / \mathrm{mL}[70,71]$. The antibacterial potency of this compound isolated from E. elephantina is noteworthy as the species is administered as a remedy by traditional healers in Botswana [40, 41], Mozambique [45], South Africa [15, 28, 29, 32, 38, 44, 46], and Swaziland [34]. These results support the traditional use of E. elephantina in treating bacterial infections such as diarrhoea and sexually transmitted infections.

7.3. Antifungal Activity. Aaku et al. [41] evaluated the antifungal activity of $70 \%$ ethanol and n-butanol rhizome extracts of E. elephantina using the TLC bioautography technique with chloramphenicol and miconazole as positive and negative controls, respectively. Both extracts showed activity against Candida mycoderma at loadings lower than $15 \mu \mathrm{g}$. These results support the traditional use of E. elephantina in treating fungal infections associated with gastrointestinal tract infections. Mukanganyama et al. [21] evaluated antifungal activities of root ethanol extracts of E. elephantina against Candida albicans and Candida mycoderma using the agar diffusion assay. The species exhibited antifungal properties against both microorganisms tested and the authors assessed the minimal inhibitory concentrations (MICs) against Candida albicans and E. elephantina showed some activity with MIC value of $1.25 \mathrm{mg} / \mathrm{mL}$ [21]. Mabona et al. [61] evaluated antifungal activities of aqueous and dichlomethane/methanol $(1: 1)$ extracts of E. elephantina using the microtitre plate dilution technique against dermatologically relevant pathogens such as Candida albicans, Microsporum canis, and Trichophyton mentagrophytes with amphotericin B as positive control and acetone and dimethyl sulfoxide (DMSO) as negative controls. Mabona et al. [61] found varied antifungal activities of the aqueous and dichlomethane/methanol (1:1) leaf, root, and rhizome extracts with minimum inhibition concentration (MIC) 0.05 to $>16.00 \mathrm{mg} / \mathrm{mL}$. Noteworthy antifungal activities were displayed by dichlomethane/methanol leaf, root, and rhizome extracts against Microsporum canis $(0.50 \mathrm{mg} / \mathrm{mL})$, Candida albicans $(1.00 \mathrm{mg} / \mathrm{mL})$, and Trichophyton mentagrophytes $(1.00 \mathrm{mg} / \mathrm{mL})$. The aqueous root extracts of Pentanisia prunelloides combined (1:1) with $E$. elephantina displayed synergistic interactions with sum of the fractional inhibitory concentration ( $\Sigma$ FIC) values ranging from 0.31 to $0.38 \mathrm{mg} / \mathrm{mL}$ against Candida albicans. The synergistic interactions noted for Pentanisia prunelloides and E. elephantina by Mabona et al. [61] validate their antifungal effects as these two species are often used in combination as herbal medicines to treat skin infections. Similarly, Nciki et al. [50] evaluated antifungal activities of aqueous and dichlomethane/methanol (1:1) root extract of E. elephantina using the microtitre plate dilution technique against dermatologically relevant pathogens such as Candida albicans, Microsporum canis, and Trichophyton mentagrophytes with amphotericin B as positive control. Best antifungal results were demonstrated by dichlomethane/methanol extracts against Candida albicans with MIC value of $130 \mu \mathrm{g} / \mathrm{mL}$, Microsporum canis (MIC value of $250 \mu \mathrm{g} / \mathrm{mL}$ ), and Trichophyton mentagrophytes with MIC value of $250 \mu \mathrm{g} / \mathrm{mL}$ [50]. It is important to note that Nciki et al. [50] assessed antifungal activities of root extracts only while Mabona et al. [61] evaluated antifungal roots, leaves, and rhizomes of E. elephantina. There are also differences in terms of best antifungal results documented in these two studies. According to Mabona et al. [61] the best antifungal activities were demonstrated by dichlomethane/methanol leaf, root, and rhizome extracts against Microsporum canis with MIC value of $0.50 \mathrm{mg} / \mathrm{mL}$ while best antifungal results obtained by Nciki et al. [50] were demonstrated by dichlomethane/methanol extracts against Candida albicans with MIC value of $130 \mu \mathrm{g} / \mathrm{mL}$. Overall, results obtained by Nciki et al. [50] and Mabona et al. [61] provide a scientific basis for the traditional use of $E$. elephantina as herbal medicine against several skin infections in South Africa such as acne [15, 36-38], eczema [36, 37], itching [12], sores [50, 51], and sunburn $[15,38]$.

7.4. Anti-Inflammatory and Antinociceptive Activities. Maphosa et al. [62] evaluated anti-inflammatory and antinociceptive activities of root extract of E. elephantina using Wistar rats. The authors evaluated anti-inflammatory activities using carrageenan and histamine-induced rat paw oedema while antinociceptive activity was evaluated by acetic acid-induced writhing test and formalin test. The aqueous extract of E. elephantina reduced the formation of oedema induced by carrageenan and histamine and caused reduction in writhings in the acetic acid test and licking time in the formalin test [62]. According to Maphosa et al. [62], the root extract of E. elephantina reduced oedema and pain even better than the control, indomethacin, a potent inhibitor of prostaglandins (PG) synthesis, showing that the plant species has strong anti-inflammatory and antinociceptive activities. The anti-inflammatory activity displayed by root extract of $E$. elephantina could be due to anthraquinone $\mathbf{3 8}$, as previous research by Mishchenko et al. [72] showed that cell culture composed of anthraquinone $\mathbf{3 8}$ isolated from Rubia cordifolia L. exhibited anti-inflammatory activity, which is manifested by an antiexudative effect and antiproliferative action during the rapid development of a model edema. These results support the traditional use of the species in various inflammatory ailments and diseases ranging from microbial infections to sores and wounds that result in cell injury and pain.

7.5. Antiplasmodial Activity. Clarkson et al. [63] evaluated aqueous, dichloromethane, and dichlomethane/methane $(1: 1)$ leaf and root extracts of E. elephantina for in vitro activity against Plasmodium falciparum using the parasite lactase dehydrogenase (pLDH) assay and chloroquine diphosphate (Sigma) as the positive control. The dichlomethane/methane $(1: 1)$ leaf and root extracts showed weak activity with $\mathrm{IC}_{50}$ values of 26 and $28 \mu \mathrm{g} / \mathrm{mL}$, respectively, while aqueous extracts for both leaves and roots showed weak activity with $\mathrm{IC}_{50}$ values $>100 \mu \mathrm{g} / \mathrm{mL}$ [62]. Although E. elephantina is widely used as traditional remedy for fever in Mozambique 
[25], South Africa [42], and Zimbabwe [43], the species did not display promising in vitro antiplasmodial activity, to support its traditional usage in the management and treatment of fever. A possible explanation could be that E. elephantina act as antipyretics or immune stimulants to relieve the symptoms of the disease, rather than having direct antiparasitic activity [73]. Alternatively, precursors of the active components may be present in E. elephantina extracts but have to be modified, usually in vivo, before activity is exhibited [63].

7.6. Antioxidant Properties. Mpofu et al. [54] evaluated antioxidant properties of E. elephantina using DPPH radical scavenging method with the yen and duh percentage inhibition values ranging from 33 to $72 \%$ for both methanol and aqueous extracts. This study carried out by Mpofu et al. [54] revealed that there were more extractable antioxidants using methanol compared to water as the solvent. The antioxidant activities demonstrated by E. elephantina rhizome extracts are probably due to the presence of flavonoids and phenolics [74]. Antioxidant properties displayed by E. elephantina could be due to the compound ethyl gallate 4 . Ethyl gallate 4 isolated from ethanol extract of Acacia nilotica Wild ex Del. subsp. indica (Benth.) Brenan leaves demonstrated antioxidant activities in several in vitro assays [75], revealing that the compound was a hydrogen donor, metal chelator, and free radical scavenger.

7.7. Antirickettsial and Antibabesial. Antibabesial and antirickettsial in vitro assay systems have been used to evaluate E. elephantina rhizome extracts. Naidoo et al. [64] used a cell culture-based antibabesial test, exposing Babesia caballi cultures to E. elephantina, and effectivity was established by the degree of inhibition using a colour change method as well as by evaluating percentage of parasitized cells on thin culture smears and calculating the degree of residual infectivity. The antibabesial drugs used as controls, imidocarb and diminazene, demonstrated efficacy, exhibiting $\mathrm{EC}_{50}$ values of 0.08 and $0.30 \mu \mathrm{g} / \mathrm{mL}$, respectively. Similarly, E. elephantina acetone rhizome extract demonstrated activity at $100 \mu \mathrm{g} / \mathrm{mL}$. Acetone rhizome extracts of E. elephantina demonstrated significant activity against a tick-borne disease that is problematic to the livestock of South African farmers [64].

Naidoo et al. [65] evaluated the antirickettsial activity of leaf acetone extracts of E. elephantina in an in vitro Ehrlichia ruminantium culture system. Ehrlichia ruminantium cultures were incubated with acetone extracts of the leaves and results were compared to those obtained with oxytetracycline and untreated controls. Elephantorrhiza elephantina possessed antiehrlichial activity with $\mathrm{EC}_{50}$ values of $111.40 \mu \mathrm{g} / \mathrm{mL}$ and $\mathrm{EC}_{90}$ values of $228.90 \mu \mathrm{g} / \mathrm{mL}$. The $\mathrm{EC}_{50}$ and $\mathrm{EC}_{90}$ values for oxytetracycline were 0.29 and $0.08 \mu \mathrm{g} / \mathrm{mL}$. These results demonstrate that E. elephantina leaf extracts may be inhibitory against the Ehrlichia parasite by a similar mechanism to each other, which was unrelated to the mechanism of action of the tetracyclines [65].

7.8. Toxicity. Despite the long use of E. elephantina as herbal medicine in southern Africa to treat numerous human and animal diseases and ailments, the species is known to be harmful when used at an excessive dosage [3, 4, 48]. Root infusions of $E$. elephantina have been reported to have constipating effects [48] while seeds are strongly irritant and have been suspected of causing human death when used as herbal medicine [4]. According to Hutchings et al. [4] an aqueous extract of the seed equivalent to $0,75 \mathrm{~g}$ produced extensive necrosis at the point of injection and gastroenteritis and pulmonary oedema when injected subcutaneously in the guinea-pig. Symptoms of poisoning were apathy, loss of appetite, and profuse foetid diarrhoea with death occurring within twenty-four hours with the animal in a state of exhaustion. Postmortem examination revealed acute gastroenteritis with numerous haemorrhages and marked degeneration of the liver [4]. Jansen [16] reported that the seeds of $E$. elephantina are toxic to sheep with a lethal dose $250 \mathrm{~g}$ and rabbits (lethal dose $5-7.50 \mathrm{~g} / \mathrm{kg}$ ) causing gastroenteritis and pulmonary oedema.

Preliminary acute toxicity evaluation of root extract of $E$. elephantina using Wistar rats showed no physiological and behavioural changes in the animals and also no mortalities were recorded [62]. In another study, Maphosa et al. [76] evaluated the acute, subacute, and chronic toxicity of E. elephantina root extracts by oral route in male and female Wistar rats. The authors recorded no mortalities but changes in body weight and haematological and serum biochemical parameters between the control and treated animals were observed. In acute tests, Maphosa et al. [76] observed decreased respiratory rate at higher doses of $1600 \mathrm{mg} / \mathrm{kg}$, and, in subacute tests, the root extract of E. elephantina caused an increase in white blood cells, monocytes, and serum levels of creatinine at higher doses of 400 and $800 \mathrm{mg} / \mathrm{kg}$. In chronic toxicity, E. elephantina extracts caused increase in lymphocytes and platelets and changes were also noted in the body and organ weights in both subacute and chronic toxicities. Maphosa et al. [76] observed acute hepatitis, intracrystal deposition (reminiscent of oxalate crystals) with renal crystals and secondary ascending pyelonephritis in animals receiving $800 \mathrm{mg} / \mathrm{kg}$ in subacute toxicity tests while pulmonary granulomas were noted in animals which received $400 \mathrm{mg} / \mathrm{kg}$. In chronic toxicity tests, Maphosa et al. [76] observed mild to moderate splenic siderosis, pulmonary granulomas, refractile crystal deposits, and associated ascending pyelonephritis. Mpofu et al. [54] evaluated cytotoxicity activity of E. elephantina using the brine shrimp lethality test. Chloroform rhizome extract of E. elephantina exhibited some degree of biological activity with $\mathrm{LC}_{50}$ value of 0.80 [54]. Based on toxicity evaluations done so far $[54,62,76]$, it can be inferred that E. elephantina has some potential toxicity at certain dose levels and should be taken with caution when used as herbal medicine.

\section{Conclusion}

The present review summarizes the ethnomedicinal uses and recent findings on traditional uses, phytochemistry, pharmacology, and toxicity of different extracts and compounds of E. elephantina. Anthocyanidins, anthraquinones, esters, fatty acids, phenolic compounds, flavonoids, glycosides, polysterols, saponins, sugars, tannins, and triterpenoids 
have been demonstrated to be the main active ingredients of E. elephantina. Recent studies have focused on evaluating anthelmintic, antibacterial, antifungal, antiinflammatory and antinociceptive, antiplasmodial, antioxidant, antibabesial, and antirickettsial activities of the different extracts and compounds isolated from the species. In the past 30 years, E. elephantina has been the subject of phytochemical and pharmacological research, and some of the traditional uses of this plant particularly against microbial infections and gastrointestinal parasites in animals have been validated by pharmacological studies. But there is not yet enough data on ethnopharmacological evaluation and clinical research on the species and few evaluations of target-organ toxicity have been documented. Most of the phytochemical and pharmacological evaluations have focused on rhizomes and roots of E. elephantina. The most important research gaps identified in this study are as follows:

(1) Since E. elephantina is widely used in combination with other plant species in various herbal concoctions, there is need for extensive research to evaluate synergistic effects of the different extracts or pure isolates to evaluate their ability to enhance the efficiency of the additive mixtures,

(2) Future research should also focus on aerial parts of the species in order to ensure full utilization of the possible medicinal potential of E. elephantina. There is need to investigate the chemical constituents and pharmacological effects of the bark, leaves, flowers, fruits, and seeds of E. elephantina.

(3) Literature studies show that the major phytochemical compounds isolated from E. elephantina so far are mainly fatty acids, phenolic compounds, and esters, but very little attempt has been made to correlate the activities of these compounds with the ethnomedicinal uses of the species. Therefore, there is need for further research on different compounds isolated from E. elephantina; examples include fatty acids and esters. Detailed phytochemical studies of E. elephantina and its pharmacological properties especially the mechanism of action of its bioactive constituents to illustrate the correlation between its ethnomedicinal uses and pharmacological activities should be the focus of future research studies.

(4) Extensive in vivo experiments are required to validate the existing pharmacological activities.

(5) Since E. elephantina contain potentially toxic compounds, future studies should include the identification of toxic compounds, possible side effects caused by taking E. elephantina as herbal medicine, and mechanisms of how potential toxic components of the species can be managed.

\section{Conflicts of Interest}

The author declares that there are no conflicts of interest regarding the publication of this paper.

\section{Acknowledgments}

The author would like to express his gratitude to the National Research Foundation (NRF) and Govan Mbeki Research and Development Centre (GMRDC), University of Fort Hare, for financial support to conduct this research.

\section{References}

[1] H. F. Glen, "Elephantorrhiza," in Plants of Southern Africa: An Annotated Checklist, G. Germishuizen and N. L. Meyer, Eds., Strelitzia 14, p. 508, National Botanical Institute, Pretoria, South Africa, 2003.

[2] E. Palmer and P. Pitman, Trees for Southern Africa Covering all Known Indigenous Species in Republic of South Africa, South West Africa, Botswana, Lesotho and Swaziland, A.A. Balkema, Cape Town, South Africa, 1972.

[3] M. Gelfand, S. Mavi, R. B. Drummond, and B. Ndemera, The Traditional Medical Practitioner in Zimbabwe. His Principles of Practice and Pharmacopoeia, Mambo Press, Gweru, Zimbabwe, 1985.

[4] A. Hutchings, A. H. Scott, G. Lewis, and A. Cunningham, Zulu Medicinal Plants. An Inventory, University of Natal Press, Pietermarizburg, South Africa, 1996.

[5] A. Maroyi, "Phytochemical and ethnopharmacological review of Elephantorrhiza goetzei (Harms) Harms," Asian Pacific Journal of Tropical Medicine, vol. 10, no. 2, pp. 107-113, 2017.

[6] M. Coates Palgrave, Keith Coates Palgrave Trees of Southern Africa, Struik Publishers, Cape Town, South Africa, 2002.

[7] M. Mander, N. Diederichs, and N. Steytler, "Marketing of Medicinal Plants and Products," in Commercialising Medicinal Plants: A Southern African Guide, pp. 167-192, Sun Press, Stellenbosch, South Africa, 2005.

[8] A. P. Dold and M. L. Cocks, "The trade in medicinal plants in the Eastern Cape Province, South Africa," South African Journal of Science, vol. 98, no. 11-12, pp. 589-597, 2002.

[9] D. F. S. Ah Goo, The contribution of the trade in medicinal plants to urban livelihoods: A case study of the informal markets In Nelson Mandela Bay Municipality, Eastern Cape [MSc dissertation], Nelson Mandela Metropolitan University, Port Elizabeth, South Africa, 2012.

[10] V. L. Williams, K. Balkwill, and E. T. F. Witkowski, "A lexicon of plants traded in the Witwatersrand umuthi shops, South Africa," Bothalia, vol. 31, no. 1, pp. 71-98, 2001.

[11] T. E. Moeng, An Investigation Into the Trade of Medicinal Plants by Muthi Shops and Street Vendors in the Limpopo Province, South Africa [MSc dissertation], University of Limpopo, Sovenga, South Africa, 2010.

[12] C. M. Monakisi, Knowledge and use of traditional medicinal plants by the setswana-speaking community of kimberley, Northern Cape of South Africa [M.S. dissertation], Stellenbosch University, Cape Town, South Africa, 2007.

[13] S. Talukdar, "Lesotho," in Southern African Plant Red Data Lists, J. S. Golding, Ed., Southern African Botanical Diversity Network Report No. 14., pp. 21-30, Sabonet, Pretoria, South Africa, 2002.

[14] B.-E. Van Wyk, "The potential of South African plants in the development of new medicinal products," South African Journal of Botany, vol. 77, no. 4, pp. 812-829, 2011.

[15] B.-E. van Wyk, B. van Oudtshoorn, and N. Gericke, Medicinal Plants of South Africa, Briza Publications, Pretoria, South Africa, 2013. 
[16] P. C. M. Jansen, "Elephantorrhiza elephantina," in Plant Resources of Tropical Africa 3: Dyes and Tannins, P. C. M. Jansen and D. Cardon, Eds., pp. 75-76, PROTA Foundation, Backhuys Publishers, Wageningen, The Netherlands, 2005.

[17] E. Schmidt, M. Lotter, and W. McCleland, Trees and Shrubs of Mpumalanga and Kruger National Park, Jacana Media, Johannesburg, South Africa, 2002.

[18] A. Maroyi, L. J. G. van der Maesen, and L. Gloriosa superba, "(Colchicaceae): Ethnobotany and economic importance," in African Plant Diversity: Systematics and Sustainable Development: Proceedings of the 19th AETFAT Congress, N. Beau, S. Dessein, and E. Robbrecht, Eds., vol. 50, pp. 408-416, Scripta Botanica Belgica, Antananarivo, Madagascar, 26-30 April 2010.

[19] O. N. Allen and E. K. Allen, The Leguminosae, University of Winsconsin Press, madison, wis, USA, 1981.

[20] A. Grobler, Elephantorrhiza elephantina, (Burch.) Skeels, National Herbarium, Pretori, 2010.

[21] S. Mukanganyama, A. N. Ntumy, F. Maher, M. Muzila, and K. Andrae-Marobela K, "Screening for anti-infective properties of selected medicinal plants from Botswana," The African Journal of Plant Science and Biotechnology, vol. 5, no. 1, pp. 1-7, 2011.

[22] J. C. Moreki, K. Tshireletso, and I. C. Okoli, "Potential use of ethnoveterinary medicine for retained placenta in cattle in Mogonono, Botswana," Journal of Animal Production Advances, vol. 2, no. 6, pp. 303-309, 2012.

[23] J. C. Moreki, "Documentation of ethnoveterinary practices used in family poultry in Botswana," Veterinary World, vol. 6, no. 1, pp. 18-21, 2013.

[24] L. S. Kose, A. Moteetee, and S. van Vuuren, "Ethnobotanical survey of medicinal plants used in the Maseru district of Lesotho," Journal of Ethnopharmacology, vol. 170, pp. 184-200, 2015.

[25] A. Ribeiro, M. M. Romeiras, J. Tavares, and M. T. Faria, "Ethnobotanical survey in Canhane village, district of Massingir, Mozambique: medicinal plants and traditional knowledge," Journal of Ethnobiology and Ethnomedicine, vol. 6, article 33, 2010.

[26] Tree Atlas of Namibia, Elephantorrhiza elephantina: Omundjoze, 2017, http://treeatlas.biodiversity.org.na/viewspec.php?nr=224.

[27] D. Luseba and D. van der Merwe, "Ethnoveterinary medicine practices among Tsonga speaking people of South Africa," Onderstepoort Journal of Veterinary Research, vol. 73, no. 2, pp. 115-122, 2006.

[28] M. C. Mathabe, R. V. Nikovola, N. Lall, and N. Z. Nyazema, "Antibacterial activities of medicinal plants used for the treatment of diarrhoea in Limpopo Province, South Africa," Journal of Ethnopharmacology, vol. 105, no. 1-2, pp. 286-293, 2006.

[29] J. R. Appidi, D. S. Grierson, and A. J. Afolayan, "Ethnobotanical study of plants used for the treatment of diarrhoea in the Eastern Cape, South Africa," Pakistan Journal of Biological Sciences, vol. 11, no. 15, pp. 1961-1963, 2008.

[30] W. Beinart and K. Brown, African Local Knowledge and Livestock Health: Diseases and Treatments in U.S, African Studies Centre, University of Oxford, Oxford, UK, 2013.

[31] S. S. Semenya, A. Maroyi, M. J. Potgieter, and L. J. C. Erasmus, "Herbal medicines used by Bapedi traditional healers to treat reproductive ailments in the Limpopo Province, South Africa," African Journal Traditional, Complementary and Alternative Medicine, vol. 10, no. 2, pp. 331-339, 2013.

[32] S. A. Rankoana, "Sustainable use and management of indigenous plant resources: a case of Mantheding community in
Limpopo Province, South Africa," Sustainability (Switzerland), vol. 8, no. 3, article 221, 2016.

[33] M. Sanhokwe, J. Mupangwa, P. J. Masika, V. Maphosa, and V. Muchenje, "Medicinal plants used to control internal and external parasites in goats," Onderstepoort Journal of Veterinary Research, vol. 83, no. 1, Article ID a1016, 7 pages, 2016.

[34] O. O. G. Amusan, "Some ethnoremedies used for HIV/AIDS and related diseases in Swaziland," The African Journal of Plant Science and Biotechnology, vol. 3, no. 1, pp. 20-26, 2009.

[35] H. A. Hyde, B. T. Wursten, P. Ballings, and M. Coates Palgrave, Elephantorrhiza elephantina (Burch.) Skeels. Flora of Zimbabwe: Species information: Elephantorrhiza elephantina, 2017, http:// www.zimbabweflora.co.zw/speciesdata/species.php?species_ $\mathrm{id}=126450$.

[36] J. Pujol, Natur Africa: The Herbalist Handbook, Jean Pujol NaturaJ Healers Foundation, Durban, South Africa, 1990.

[37] T. Felhaber, South African Traditional Healers' Primary Health Care Handbook, Kagiso, Cape Town, South Africa, 1997.

[38] N. Lall and N. Kishore, "Are plants used for skin care in South Africa fully explored?" Journal of Ethnopharmacology, vol. 153, no. 1, pp. 61-84, 2014.

[39] A. Jacot Guillarmod, Flora of Lesotho, Cramer, Lehr, Germany, 1971.

[40] I. Hedberg and F. Staugard, Traditional Medicinal Plants: Traditional Medicine in Botswana, lpeleng, Gabarone, Botswana, 1989.

[41] E. Aaku, M. Office, S. P. Dharani, R. R. T. Majinda, and M. S. Motswaiedi, "Chemical and antimicrobial studies on Elephantorrhiza elephantina," Fitoterapia, vol. 69, no. 5, pp. 464-465, 1998.

[42] J. Gerstner, "A preliminary check list of Zulu names of plants," Bantu Studies, vol. 13, no. 1, pp. 131-149, 1939.

[43] S. Mpofu, D. Tantoh Ndinteh, S. F. van Vuuren, D. K. Olivier, and R. W. M. Krause, "Interactive efficacies of Elephantorrhiza elephantina and Pentanisia prunelloides extracts and isolated compounds against gastrointestinal bacteria," South African Journal of Botany, vol. 94, pp. 224-230, 2014.

[44] M. A. Bisi-Johnson, C. L. Obi, L. Kambizi, and M. Nkomo, "A survey of indigenous herbal diarrhoeal remedies of O.R. Tambo district, Eastern Cape Province, South Africa," African Journal of Biotechnology, vol. 9, no. 8, pp. 1245-1254, 2010.

[45] S. O. Bandeira, F. Gaspar, and F. P. Pagula, "African ethnobotany and healthcare: emphasis on Mozambique," Pharmaceutical Biology, vol. 39, no. 1, pp. 70-73, 2001.

[46] A. T. Bryant, Zulu Medicine and Medicine-Men, C. Struik, Cape Town, South Africa, 1966.

[47] S. S. Semenya and M. J. Potgieter, "Ethnobotanical survey of medicinal plants used by Bapedi traditional healers to treat erectile dysfunction in the Limpopo Province, South Africa," Journal of Medicinal Plants Research, vol. 7, no. 7, pp. 349-357, 2013.

[48] J. M. Watt and M. G. Breyer-Brandwijk, The Medicinal and Poisonous Plants of Southern and Eastern Africa: Pharmacological Effects and Toxicology in Man and Animals, E. Livingstone, Edinburgh, UK.

[49] S. S. Semenya, M. J. Potgieter, and L. J. C. Erasmus, "Ethnobotanical survey of medicinal plants used by Bapedi healers to treat diabetes mellitus in the Limpopo Province, South Africa," Journal of Medicinal Plants Research, vol. 7, no. 8, pp. 434-441, 2013. 
[50] S. Nciki, S. Vuuren, A. van Eyk, and H. de Wet, "Plants used to treat skin diseases in northern Maputaland, South Africa: antimicrobial activity and in vitro permeability studies," Pharmaceutical Biology, vol. 54, no. 11, pp. 2420-2436, 2016.

[51] H. de Wet, S. Nciki, and S. F. van Vuuren, "Medicinal plants used for the treatment of various skin disorders by a rural community in northern Maputaland, South Africa," Journal of Ethnobiology and Ethnomedicine, vol. 9, article 51, 2013.

[52] D. van der Merwe, G. E. Swan, and C. J. Botha, "Use of ethnoveterinary medicinal plants in cattle by Setswana-speaking people in the Madikwe area of the North West Province of South Africa," Journal of the South African Veterinary Association, vol. 72, no. 4, pp. 189-196, 2001.

[53] A. P. Dold and M. L. Cocks, "Traditional veterinary medicine in the Alice district of the Eastern Cape Province, South Africa," South African Journal of Science, vol. 97, no. 9-10, pp. 375-379, 2001.

[54] S. J. Mpofu, T. A. M. Msagati, and R. W. M. Krause, "Cytotoxicity, phytochemical analysis and antioxidant activity of crude extracts from rhizomes of Elephantorrhiza elephantina and Pentanisia prunelloides," African Journal of Traditional, Complementary, and Alternative Medicines, vol. 11, no. 1, pp. 3452, 2014.

[55] H. Z. Msimanga, J. Fenstermacher, A. Levitz, I. Najimudeen, C. Phillips, and E. M. Wysocki, "Identification of compounds in hexane extracts of Elephantorrhiza elephantina and their comparison with selected over the counter products," Journal of Medicinal Plants Research, vol. 7, no. 5, pp. 198-208, 2013.

[56] S. J. Mpofu, T. A. M. Msagati, and R. W. M. Krause, "Flavonoids from the rhizomes of Elephantorrhiza elephantina and Pentanisia prunelloides," Journal of Medicinal Plants Research, vol. 9, no. 16, pp. 531-549, 2015.

[57] X. S. Mthembu, A phytochemical study of Scheflera umbellifera and Elephantorrhiza elephantina [M.S. Dissertation], University of Kwazulu Natal, Pietermaritzburg, South Africa, 2007.

[58] V. Maphosa, P. J. Masika, E. S. Bizimenyera, and J. N. Eloff, “Invitro anthelminthic activity of crude aqueous extracts of Aloe ferox, Leonotis leonurus and Elephantorrhiza elephantina against Haemonchus contortus," Tropical Animal Health and Production, vol. 42, no. 2, pp. 301-307, 2010.

[59] V. Maphosa and P. J. Masika, "In vivo validation of Aloe ferox (Mill). Elephantorrhiza elephantina Bruch. Skeels. and Leonotis leonurus (L) R. BR as potential anthelminthics and antiprotozoals against mixed infections of gastrointestinal nematodes in goats," Parasitology Research, vol. 110, no. 1, pp. 103-108, 2012.

[60] V. Maphosa and P. J. Masika, "Anthelmintic screening of fractions of Elephantorrhiza elephantina root extract against Haemonchus contortus," Tropical Animal Health and Production, vol. 44, no. 1, pp. 159-163, 2012.

[61] U. Mabona, A. Viljoen, E. Shikanga, A. Marston, and S. van Vuuren, "Antimicrobial activity of southern African medicinal plants with dermatological relevance: from an ethnopharmacological screening approach, to combination studies and the isolation of a bioactive compound," Journal of Ethnopharmacology, vol. 148, no. 1, pp. 45-55, 2013.

[62] V. Maphosa, P. J. Masika, and B. Moyo, "Investigation of the antiinflammatory and antinociceptive activities of Elephantorrhiza elephantina (Burch.) Skeels root extract in male rats," African Journal of Biotechnology, vol. 8, no. 24, pp. 7068-7072, 2009.

[63] C. Clarkson, V. J. Maharaj, N. R. Crouch et al., "In vitro antiplasmodial activity of medicinal plants native to or naturalised in
South Africa," Journal of Ethnopharmacology, vol. 92, no. 2-3, pp. 177-191, 2004.

[64] V. Naidoo, E. Zweygarth, J. N. Eloff, and G. E. Swan, "Identification of anti-babesial activity for four ethnoveterinary plants in vitro," Veterinary Parasitology, vol. 130, no. 1-2, pp. 9-13, 2005.

[65] V. Naidoo, E. Zweygarth, and G. E. Swan, "Determination and quantification of the in vitro activity of Aloe marlothii (A. Berger) subsp. marlothii and Elephantorrhiza elephantina (Burch.) skeels acetone extracts against Ehrlichia ruminantium," Onderstepoort Journal of Veterinary Research, vol. 73, no. 3, pp. 175-178, 2006.

[66] C. Cueva, S. Mingo, I. Muñoz-González et al., "Antibacterial activity of wine phenolic compounds and oenological extracts against potential respiratory pathogens," Letters in Applied Microbiology, vol. 54, no. 6, pp. 557-563, 2012.

[67] F. A. Hashem and M. M. Saleh, "Antimicrobial components of some cruciferae plants (Diplotaxis harra Forsk. and Erucaria microcarpa Boiss.)," Phytotherapy Research, vol. 13, no. 4, pp. 329-332, 1999.

[68] J. Yang, X. Hou, P. S. Mir, and T. A. McAllister, "Anti-Escherichia coli O157:H7 activity of free fatty acids under varying $\mathrm{pH}$," Canadian Journal of Microbiology, vol. 56, no. 3, pp. 263-267, 2010.

[69] M. Abhilash, "In silico analysis of cranberry proanthocyanidin epicatechin (4beta-8, 2beta-0-7) as an inhibitor for modelled afimbrial adhesin virulence protein of uropathogenic Escherichia coli," International Journal of Pharma and Biological Sciences, vol. 1, no. 1, pp. 1-7, 2010.

[70] R. Krause, E. Schwab, D. Bachhiesl et al., "Role of Candida in antibiotic-associated diarrhea," Journal of Infectious Diseases, vol. 184, no. 8, pp. 1065-1069, 2001.

[71] A. Jouret-Mourin and K. Geboes, "Infectious colitis," Acta Endoscopica, vol. 32, no. 2, pp. 167-183, 2002.

[72] N. P. Mishchenko, S. A. Fedoreev, V. M. Bryukhanov et al., "Chemical composition and pharmacological activity of anthraquinones from Rubia cordifolia cell culture," Pharmaceutical Chemistry Journal, vol. 41, no. 11, pp. 605-609, 2007.

[73] J. D. Phillipson, C. W. Wright, G. C. Kirby, and D. C. Warhurst, "Tropical plants as sources of antiprotozoal agents," in Phytochemical Potential of Tropical Plants, K. R. Downum, J. T. Romeo, and H. A. Stafford, Eds., pp. 1-40, Plenum Press, New York, NY, USA, 1993.

[74] A. R. Ndhlala, C. Mupure, K. Chitindingu et al., "Antioxidant potentials and degrees of polymerization of six wild fruits," Scientific Research and Essay, vol. 1, no. 3, pp. 87-92, 2006.

[75] T. Kalaivani, C. Rajasekaran, and L. Mathew, "Free radical scavenging, cytotoxic, and hemolytic activities of an active antioxidant compound ethyl gallate from leaves of Acacia Nilotica(L.) wild. ex. delile subsp. Indica (Benth.) Brenan," Journal of Food Science, vol. 76, no. 6, pp. T144-T149, 2011.

[76] V. Maphosa, P. J. Masika, and B. Moyo, “Toxicity evaluation of the aqueous extract of the rhizome of Elephantorrhiza elephantina (Burch.) Skeels. (Fabaceae), in rats," Food and Chemical Toxicology, vol. 48, no. 1, pp. 196-201, 2010. 


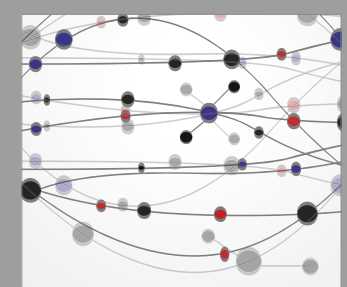

The Scientific World Journal
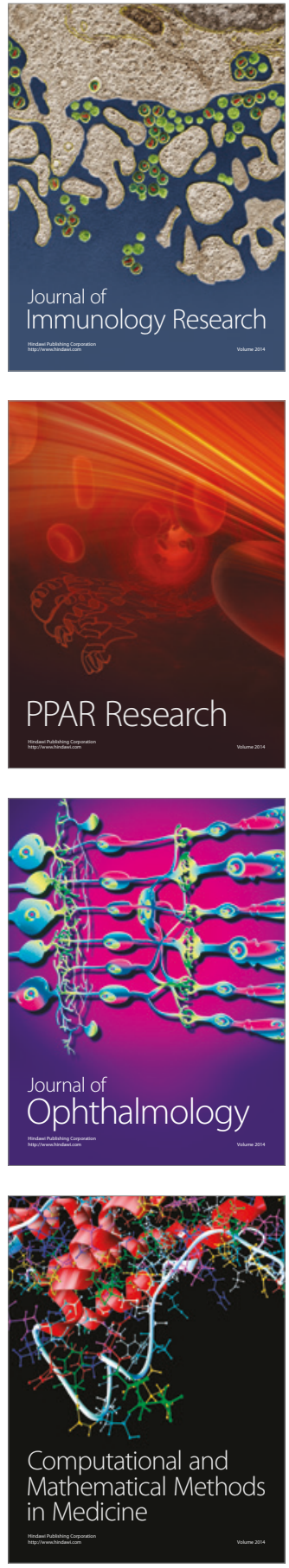

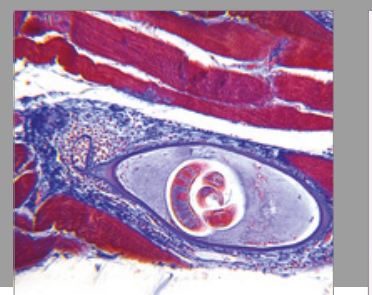

Gastroenterology Research and Practice
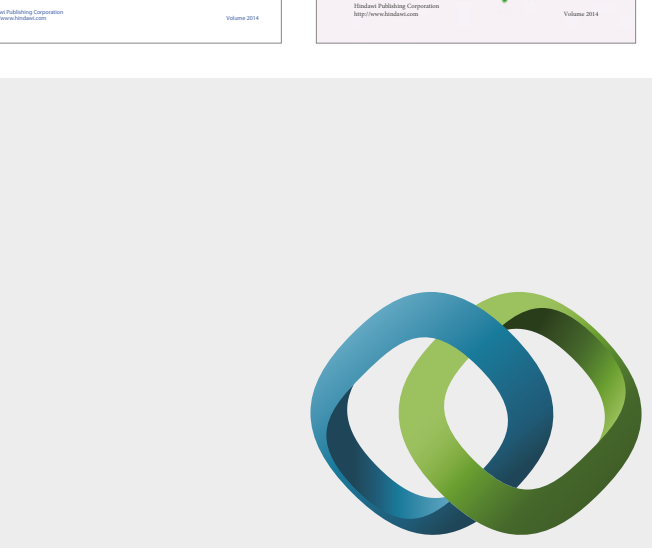

\section{Hindawi}

Submit your manuscripts at

https://www.hindawi.com
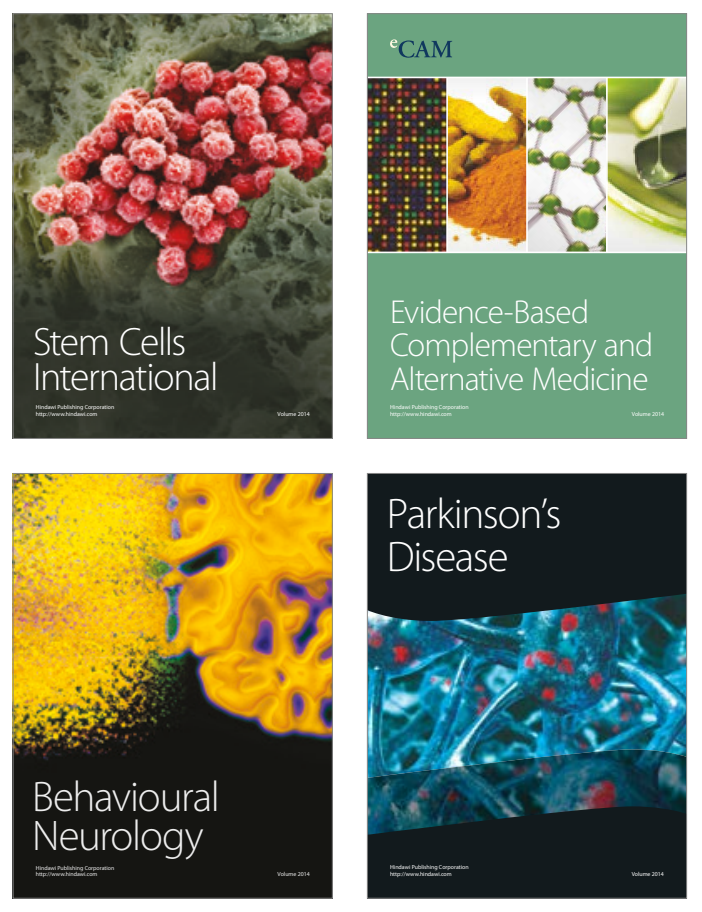
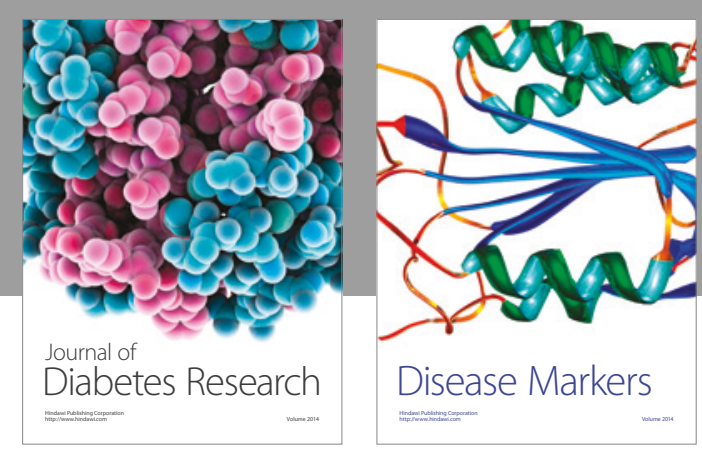

Disease Markers
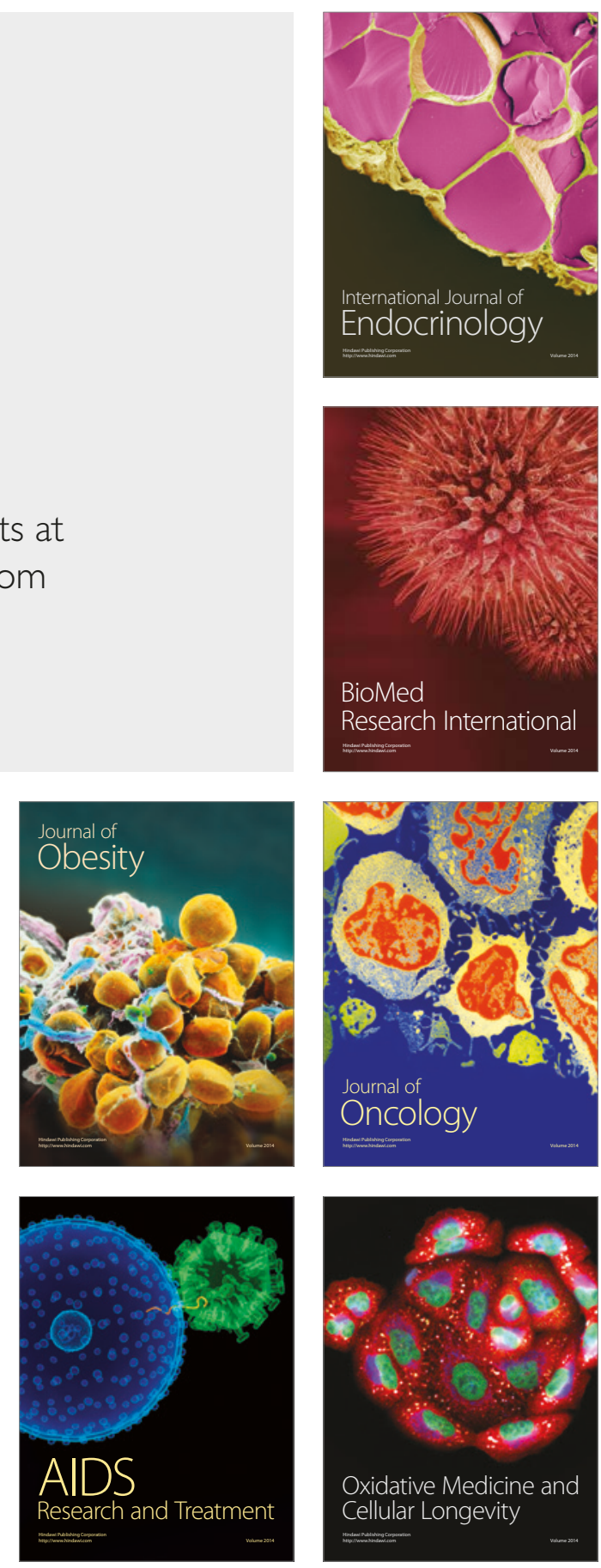